\title{
Photoreceptor Neurons Find New Synaptic Targets When Misdirected by Overexpressing runt in Drosophila
}

\author{
Tara N. Edwards ${ }^{1}$ and Ian A. Meinertzhagen ${ }^{1,2}$ \\ Departments of 1Biology and 2Psychology, Life Sciences Centre, Dalhousie University, Halifax, Nova Scotia, Canada B3H 4J1
}

\begin{abstract}
As a neuron differentiates, it adopts a suite of features specific to its particular type. Fly photoreceptors are of two types: R1-R6, which innervate the first optic neuropile, the lamina; and R7-R8, which innervate the second, the medulla. Photoreceptors R1-R6 normally have large light-absorbing rhabdomeres, express Rhodopsin1, and have synaptic terminals that innervate the lamina. In Drosophila melanogaster, we used the yeast GAL4/UAS system to drive exogenous expression of the transcription factor Runt in subsets of photoreceptors, resulting in aberrant axonal pathfinding and, ultimately, incorrect targeting of R1-R6 synaptic terminals to the medulla, normally occupied by terminals from R7 and R8. Even when subsets of their normal R1-R6 photoreceptor inputs penetrate the lamina, to terminate in the medulla, normal target cells within the lamina persist and maintain expression of cell-specific markers. Some R1-R6 photoreceptors form reciprocal synaptic inputs with their normal lamina targets, whereas supernumerary terminals targeted to the medulla also form synapses. At both sites, tetrad synapses form, with four postsynaptic elements at each release site, the usual number in the lamina. In addition, the terminals at both sites are invaginated by profiles of glia, at organelles called capitate projections, which in the lamina are photoreceptor sites of vesicle endocytosis. The size and shape of the capitate projection heads are identical at both lamina and medulla sites, although those in the medulla are ectopic and receive invaginations from foreign glia. This uniformity indicates the cellautonomous determination of the architecture of its synaptic organelles by the presynaptic photoreceptor terminal.
\end{abstract}

Key words: Drosophila melanogaster; medulla; synaptogenesis; axonal targeting; capitate projection; photoreceptor

\section{Introduction}

Development of the nervous system requires that neurons not only find their correct targets in the brain but also form correct synaptic partnerships once they contact those targets. Many studies focus on either aspect of neural development, but few consider both in a single paradigm. Their strictly ordered topography (Kaas, 1997; Chklovskii and Koulakov, 2004) and development (McLaughlin and O'Leary, 2005), as well as regulated synaptic composition (Nicol and Meinertzhagen, 1982; Rao-Mirotznik et al., 1995), make visual systems ideally suited to study both pathfinding and synaptogenesis. To navigate to their targets in the developing brain, axons in both vertebrate and invertebrate visual systems use related guidance molecules, such as receptor protein tyrosine kinases (Dütting et al., 1999) and phosphatases

\footnotetext{
Received March 7, 2008; revised Nov. 28, 2008; accepted Dec. 6, 2008.

This work was supported by a Natural Sciences and Engineering Research Council (0ttawa) postgraduate scholarship (T.N.E.) and by National Institutes of Health Grant EY-03592 (I.A.M.). Antibodies $24 B 10$ and 6D6 developed by Seymour Benzer, 7E8A10 by Gerald M. Rubin, 40-1a by Joshua Sanes, nc 82 by Erich Buchner, 1 D4 by Corey Goodman, MR1A by Chris Doe, and 4C5 by Heinz Gert de Couet and Teiichi Tanimura were obtained from the Developmental Studies Hybridoma Bank (University of lowa, lowa City, IA). Anti-Repo was provided by Dr. J. Urbin (Gutenberg Universität, Mainz, Germany), and anti-BOSS and anti-BSH by Dr. Larry Zipursky [University of California, Los Angeles (UCLA), Los Angeles, CA]. MT14-GAL4 and UAS-runt stocks were generously provided by Dr. Utpal Banerjee (UCLA), and UAS-HRP::CD2 and neoFRT lines for MARCM crosses by Dr. Chi-Hon Lee (National Institutes of Health, Bethesda, MD). All other stocks were provided by the Bloomington Stock Center. We thank Zhiyuan Lu and Rita Kostyleva for assistance with EM techniques and sectioning, Dr. Claudia Groh for comments on this manuscript, and Dr. Chi-Hon Lee for advice.

Correspondence should be addressed to lan A. Meinertzhagen, Life Sciences Centre, Dalhousie University, Halifax, Nova Scotia, Canada B3H 4J1. E-mail: iam@dal.ca.

DOI:10.1523/JNEUROSCI.1022-08.2009

Copyright $\odot 2009$ Society for Neuroscience $\quad 0270-6474 / 09 / 290828-14 \$ 15.00 / 0$
}

(Johnson et al., 2001). Related events also occur in adult organization. Thus, when photoreceptors degenerate in the vertebrate retina, reactive changes occur to form ectopic synapses between novel partners (Peng et al., 2000, 2003; Johnson et al., 2006; Bayley and Morgans, 2007). In the visual system of Drosophila, we can study these phenomena by genetic interventions, without invoking cell degeneration, but instead by targeting photoreceptor axons to an incorrect neuropile in the brain.

The visual system of Drosophila is remarkable for its numerical and spatial determinacy, especially at its identified photoreceptor synapses (Meinertzhagen and Hanson, 1993; Prokop and Meinertzhagen, 2006). The eye has two types of photoreceptors: R1-R6, which terminate in the first optic neuropile, the lamina, can be considered equivalent to vertebrate rods; whereas R7 and $\mathrm{R} 8$, with axons that terminate in different strata of the second neuropile, or medulla, are equivalent to cones. The lamina is thus formally equivalent to the outer plexiform layer of the retina, and responsible for contrast encoding (Laughlin et al., 1987), whereas the medulla assumes many of the functions of the inner plexiform layer.

Photoreceptor axons in the fly's visual system undergo morphogenesis in three stages (Meinertzhagen and Hanson, 1993; Hiesinger et al., 2006). In the initial stage, axonal pathfinding, interactions between the ingrowing photoreceptor axons and glia in the developing brain (Chotard and Salecker, 2004; Freeman, 2006) play a major role in ensuring that the axons first target their correct neuropile. This is followed by lateral targeting, during which axons find their correct synaptic partners (Meinertzhagen 
and Hanson, 1993). Photoreceptor synapses then assemble element by element, when dendrites from lamina cell targets converge on presynaptic sites to form the postsynaptic tetrads of the adult (Fröhlich and Meinertzhagen, 1982). Each R1-R6 terminal forms $\sim 50$ evenly dispersed tetrads (Meinertzhagen and Sorra, 2001). Correct retinotopic targeting of photoreceptors is regulated by many genes (Mast et al., 2006) and is independent of neuronal activity (Hiesinger et al., 2006). With these features as a basis, what then happens to R1-R6 photoreceptors that fail to terminate in the lamina and are genetically mistargeted to the medulla? Our study examines whether these photoreceptors still form synapses in the lamina, through which their axons must pass, and whether supernumerary photoreceptor terminals synapse with new partners in the medulla.

\section{Materials and Methods \\ Fly strains}

Fruit flies, Drosophila melanogaster, were raised on standard cornmeal molasses medium at $23^{\circ} \mathrm{C}$ for all crosses unless otherwise noted. The wild-type stock was Oregon R (OR). We used the GAL4/UAS system (Brand and Perrimon, 1993) to construct flies in which the R1-R6 photoreceptors bypass the lamina and mistarget to the medulla. For this, two GAL4 lines were used to drive UAS-runt (Dormand and Brand, 1998) expression: GMR-GAL4 (Moses and Rubin, 1991; Freeman, 1996), which drives expression in all photoreceptors, and MT14-GAL4 (Tissot et al., 1997). The MT14-GAL4 and UAS-runt lines were provided by Dr. Utpal Banerjee (University of California, Los Angeles, Los Angeles, CA).

We distinguished subsets of R7 and R8 photoreceptors using the R7 rhodopsin (Rh)-specific expression lines $\left.w^{*}\right] ; \quad c n[1] b w[1] / C y O$; $\mathrm{P}\{w[+m C]=R h 3$-lacZ.PD $\} 3 / T M 2$, and $w\left[^{*}\right] ; \mathrm{P}\{w[+m C]=R h 4$-lac$Z . P D\} 2 ; M K R S / T M 2$, and the R8 rhodopsin expression lines: $y[1] w\left[^{*}\right]$; $c n[1] b w[1] / C y O ; \quad \mathrm{P}\{w[+m C]=$ Rh6-lacZ.PD $\}$ 3/TM2, and $w\left[^{*}\right]$; $c n[1] b w[1] / C y O ; \mathrm{P}\{w[+m C]=R h 5-l a c Z . P D\} 3 / T M 2$. To identify Rhodopsin1 (Rh1)-expressing cells in the eye, as well as to distinguish the ectopic terminals of R1-R6 in the medulla from the terminals of R7 and $\mathrm{R} 8$, we used the $\mathrm{P}\{r y[+\mathrm{t} 7.2]=R h 1(-252 /+67)$-lacZ.omSMB $\}$ line, which expresses $\beta$-gal in R1-R6. We also used $y[1] w\left[{ }^{*}\right]$; Pin $[Y t] / C y O$; $\mathrm{P}\{U A S-m C D 8:: G F P . L\} L L 6$ to monitor GAL4 driver expression. The requisite stocks were all from the Bloomington Stock Center (Indiana University, Bloomington, IN).

A mosaic analysis with a repressible cell marker (MARCM) technique (Lee and Luo, 1999) was used to visualize individual photoreceptors in which both UAS-runt and UAS-mCD8::GFP expression was driven by GMR-GAL4. To do this, we crossed hsFLP; NeoFRT40A actin-GAL80/ CyO to UAS-mCD8::GFP; NeoFRT40A, GMR-GAL4, UAS-runt lines and selected for hsFLP/UAS-mCD8::GFP; NeoFRT40A actin-GAL80/ NeoFRT40A, GMR-GAL4, UAS-runt adult flies. Flies were reared at $23^{\circ} \mathrm{C}$ and third-instar larvae were heat shocked for $5 \mathrm{~min}$ at $37^{\circ} \mathrm{C}$.

To visualize the profiles of photoreceptor terminals by electron microscopy (EM), we used $y w$; UAS-HRP::CD2/CyO and UAS-HRP::CD2 (on III) (Larsen et al., 2003). To increase expression of the $U A S-H R P:: C D 2$ enzymatic marker, we transferred flies to $29^{\circ} \mathrm{C}$ during early pupal development or at least $24 \mathrm{~h}$ before dissection (when using GMR-GAL4 to drive UAS-runt expression). At such elevated temperatures, the UAS-runt/+; MT14-GAL4/+ flies exhibited a genetically induced motor defect, so that most failed to eclose. As representative adults, we therefore used occasional young escaper flies that emerged naturally. Otherwise, UAS-runt/+; MT14-GAL4/+, and GMR-GAL4/ UAS-runt flies were raised at $18^{\circ} \mathrm{C}$ to reduce the severity of the Runt overexpression phenotype. UAS-HRP::CD2 lines were provided by Dr. Chi-Hon Lee (National Institutes of Health, Bethesda, MD).

\section{Immunocytochemistry}

The brains of larvae and the heads of pupal and adult flies were fixed in a solution of $4 \%$ formaldehyde [as paraformaldehyde (PFA)] in $0.1 \mathrm{M}$ phosphate buffer $(\mathrm{PB})$ for $4 \mathrm{~h}$ or overnight at $4^{\circ} \mathrm{C}$. Pupal and adult brains were washed in $0.1 \mathrm{M} \mathrm{PB}$, mounted in $7 \%$ agarose, and sliced at $80-100$ $\mu \mathrm{m}$ thickness in the horizontal plane by means of a Vibratome. Brains were permeabilized in successive treatments of $0.2 \%$ Triton $\mathrm{X}(\mathrm{Tx})$ in $0.01 \mathrm{M}$ PBS and 2\% PBS-Tx, and were then blocked with 5\% normal goat serum (NGS) in $0.2 \%$ PBS-Tx. Tissues were incubated overnight at $4^{\circ} \mathrm{C}$ in antibody diluted in 5\% NGS-PBS-Tx. The following primary antibodies were used: 1:50 anti-prospero, MR1A (Spana and Doe, 1995), 1:50 mouse anti-Chaoptin, 24B10 (Zipursky et al., 1984; Van Vactor et al., 1988); 1:50 rat anti-Elav, 7E8A10 (Robinow and White, 1991; Koushika et al., 1996); 1:100 mouse anti-cysteine string protein (CSP), 6D6 (Zinsmaier et al., 1994); 1:10 anti-Fasciclin II (FasII), 1D4 (Grenningloh et al., 1991); 1:20 or 1:50 nc82 [anti-Bruchpilot (Kittel et al., 2006; Wagh et al., 2006)] and 1:50 anti- $\beta$-gal, 40-1a, all from Developmental Studies Hybridoma Bank; 1:500 rabbit anti-Repo (Campbell et al., 1994; Halter et al., 1995); 1:400 guinea pig anti-brain specific homeobox (BSH) (Jones and McGinnis, 1993); 1:100 mouse anti-BOSS (Cagan et al., 1992), 1:1000 rabbit anti-green fluorescent protein (GFP) (Invitrogen), and 1:100 rabbit anti- $\beta$-gal (Molecular Probes). The immunogen against which each antibody was raised and information on the characterization of antibody specificity are both given in supplemental Table 1 (available at www.jneurosci.org as supplemental material). After six washes in $0.2 \%$ PBS-Tx, we used one of the following single or combined secondary antibodies in 5\% NGS: FITC goat anti-mouse, Cy5 goat anti-rabbit, Cy3 goat anti-rat, $\mathrm{Cy} 3$ goat anti-mouse, $\mathrm{Cy} 3$ goat anti-rabbit, $\mathrm{Cy} 3$ goat antiguinea pig (all from Jackson ImmunoResearch); and Alexa 488 goat anti-mouse or Alexa 488 goat anti-rabbit (Invitrogen) at a concentration of either 1:200 or 1:400, and washed at least six times in PBS before being mounted in Vectashield medium (Vector Laboratories). Images were captured for confocal microscopy with either an LSM 410 or 510 instrument (Zeiss). Images were edited for publication with Adobe Photoshop CS2.

\section{Electron microscopy and histology}

The heads of adult flies were removed and bisected in a cacodylatebuffered PFA and glutaraldehyde fixative, and processed for EM, as previously described (Meinertzhagen and O'Neil, 1991). To examine retinas, tissue embedded in PolyBed 812 (catalog \#08792-1; Polysciences) was sectioned at $1.0 \mu \mathrm{m}$ and stained with a $1 \%$ toluidine blue, $1 \%$ borate solution at $60^{\circ} \mathrm{C}$, rinsed with $\mathrm{H}_{2} \mathrm{O}$, and then examined by light microscopy (Zeiss Axiophot) using a $40 \times / 0.75$ Plan Neofluar objective. Images were captured with a Zeiss AxioCam MRc 5 camera and Zeiss AxioVision imaging software. For EM, $60 \mathrm{~nm}$ sections of the optic lobe were collected, stained in uranyl acetate and lead citrate, and then examined and compared with sections from similarly prepared wild-type lamina and medulla tissue.

To examine photoreceptor axons in the medulla, MT14-GAL4 and GMR-GAL4 with or without UAS-runt were crossed to the UAS-HRP::CD2 reporter line to drive expression of horseradish peroxidase (HRP) at the plasma membrane (Larsen et al., 2003). Sites of HRP expression were confirmed from an electron-dense precipitate formed in the presence of 3,3'-diaminobenzidine (DAB) (Graham and Karnovsky, 1966; Larsen et al., 2003). Heads were fixed on ice in $4 \%$ PFA and $0.5 \%$ glutaraldehyde in $0.1 \mathrm{~m} \mathrm{~PB}$. For increased penetration of DAB, brains were either dissected out during fixation or after fixation heads were sliced at $100 \mu \mathrm{m}$ using a Vibratome. After two washes in PB, brains were treated for $20 \mathrm{~min}$ with fresh $1 \%$ sodium borohydride in $0.01 \mathrm{M}$ PBS followed by four washes in $0.01 \mathrm{~m}$ PBS. DAB solution was prepared from tablets (catalog \#D5905: Sigma-Aldrich) at a concentration of 0.2-0.5 $\mathrm{mg} / \mathrm{ml}$ with $6 \mathrm{mg} / \mathrm{ml}$ nickel ammonium sulfate in $0.01 \mathrm{M}$ Tris-buffered saline, $\mathrm{pH}$ 7.6. Brains were incubated in filtered DAB solution at least 30 min before adding $0.03 \% \mathrm{H}_{2} \mathrm{O}_{2}$ at a final concentration of $3-6 \times 10^{-6}$ $\mathrm{v} / \mathrm{v}$. Incubation times in reactive DAB varied up to $1 \mathrm{~h}$, after which brains were washed three times in TBS and postfixed in $0.5 \%$ osmium tetroxide (catalog \#19150; Electron Microscopy Sciences) in veronal acetate buffer for 30 min, dehydrated, embedded in PolyBed 812, and sectioned as before. Sections were viewed at $80 \mathrm{kV}$ in a Philips Tecnai 12 electron microscope.

Measurements and analysis were performed with software (NIH ImageJ). Per fly, at least 80 profiles of capitate projections were measured that contained the diameter of the capitate projection head, with the membranes of the photoreceptor and glial membranes clearly delineated 
and the glial core clearly visible. Measurements were made from at least two flies of each representative genotype, except for those from the R7 and $\mathrm{R} 8$ terminals in wild-type medulla, which were measured through the depth of three columns from a single fly.

\section{Results}

Drosophila mutants provide many examples of aberrant photoreceptor pathfinding. Photoreceptor axons often bypass their target neuropile if (1) they are unable to detect their target, (2) the target fails to form correctly and/or provide "stop" signals to ingrowing photoreceptors, or (3) because they are unable to defasciculate from pioneering axons when traversing the target neuropile (Mast et al., 2006). Photoreceptors growing into the lamina release two signals, Hedgehog and the EGF (epidermal growth factor)-like ligand Spitz, that result in the final mitotic division and differentiation of lamina neurons (Selleck et al., 1992; Huang and Kunes, 1996, 1998; Huang and Kunes, 1996, 1998; Huang et al., 1998). Given that R1-R6 normally terminate in the lamina, we first wondered whether R1-R6 axons were required to terminate there to enable their correct synaptic partners to continue to differentiate, or whether their target neurons would develop normally even when the R1-R6 photoreceptor axons bypass them to terminate in the medulla.

\section{Lamina neurons maintain their fates even when R1-R6 photoreceptors mistarget to the medulla}

As previously shown (Kaminker et al., 2002), axons of R1-R6 photoreceptors that express the transcription factor runt bypass the lamina and establish terminals in the underlying medulla that persist into adulthood. We used the yeast GAL4/UAS system to drive exogenous expression of runt in subsets of photoreceptors, resulting in aberrant axonal pathfinding and, ultimately, incorrect synaptic targeting. Immunocytochemical analysis of nuclear Elav reveals that when R1-R6 photoreceptor axons mistarget to the medulla, lamina monopolar neurons are nevertheless induced to form, and that these then persist into adulthood (Fig. $1 A-F)$. To identify the structure and location of cells in the optic lobe relative to the mistargeted R1-R6 axons, we used various markers to analyze successive developmental stages, from the third-instar larva to the adult.

In wild-type larvae, the R1-R6 axons terminate in the lamina plexus, between layers of epithelial and marginal glia (Poeck et al., 2001), whereas runt-expressing R7-R8 axons terminate in the medulla (Fig. 1A). When Runt is overexpressed in all photoreceptors using the GMR-GAL4 driver, many photoreceptor axons bypass the lamina and terminate in the medulla (Kaminker et al., 2002) (Fig. 1B). This transformation in R1-R6 axon trajectories enables us to examine the effects of mistargeting axons to new synaptic partners.

In the wild-type pupa (Fig. 1C), R1-R6 growth cones form a distinct lamina plexus between layers of lamina glia and below the Elav-immunoreactive nuclei of lamina monopolar neurons (L1L5) (Robinow and White, 1991). In developing GMR-GAL4/ UAS-runt flies, the lamina plexus is absent. Occasionally, the medulla fails to rotate to its normal position, with columns lying parallel to the retinal cornea, as demonstrated in a $\mathrm{P}+40 \%$ pupa (Fig. $1 D$ ), and in many cases the axons innervating the medulla completely fail to rotate by adult eclosion (data not shown). Despite the failure of the medulla to rotate, and the absence of a clear lamina plexus, neuronal nuclei, presumed to be those of L1-L5, are compressed between en passant photoreceptor axons. In both adult OR and Runt-overexpressing flies, we can distinguish layers of glia and monopolar cells in the lamina cortex (Fig. $1 E, F$ ).
Runt overexpression also affects the structure of the retina in the second half of pupal metamorphosis. At $\mathrm{P}+40 \%, \sim 48 \mathrm{~h}$ into metamorphosis, the developing retina looks relatively normal in horizontal sections (Fig. $1 D$ ). By $\mathrm{P}+60 \%$, it is apparent that the ommatidia have failed to elongate (data not shown); some ommatidia remain in a proximal location, whereas others appear to rise above their neighbors so that by eclosion the eyes are severely disrupted. From longitudinal sections of the adult retina (Fig. $1 F$ ) and observation of the surface of the eye, the retina is smaller than that of wild type and lacks distinct facets. This reduction in eye size may be responsible for the apparent reduction in the size of the lamina, given that the number of ommatidia in the fly's eye corresponds to the number of cartridges in the lamina (Braitenberg, 1967).

Expression of lamina monopolar cell-specific proteins, such as BSH in L5 (Poeck et al., 2001) and FasII in L1 and L3, reveals that these protein markers continue to be expressed despite the mistargeting of photoreceptors (Fig. $1 G-J$ ). Their expression suggests that cell fate is properly established and maintained in at least three subtypes of monopolar cells and possibly others. In mutant flies, the arrangement of proximally located $\mathrm{BSH}$-labeled L5 neurons is disordered and there appear to be fewer cells overall, presumably because of a reduction in lamina size. FasII expression is also maintained in $\mathrm{L} 1$ and $\mathrm{L} 3$ at least up to $\mathrm{P}+60 \%$ pupal development (Fig. $1 \mathrm{I}, \mathrm{J}$ ), after which time protein expression is downregulated (Hiesinger et al., 1999). Although expression of neuron-specific markers Elav, Dachshund (Mardon et al., 1994; Huang and Kunes, 1996), and BSH has been reported in the lamina cells of pathfinding mutants (Hing et al., 1999; Kaminker et al., 2002; Choe et al., 2006), such expression has previously been examined shortly after lamina cell differentiation, in the third-instar larva. Here, we report evidence that the fates of these neurons are maintained through development and that the cells persist in the adult lamina.

\section{Photoreceptors in the retinas of runt overexpression flies adopt alternative fates}

Cross-sectioned ommatidia in semithin sections of UAS-runt/+; MT14-GAL4/+ retinas clearly reveal that after Runt overexpression ommatidia occasionally have a normal complement of eight photoreceptor neurons. As seen in the wild-type fly, the large rhabdomeres of photoreceptors R1-R6 form an outer trapezoidal pattern, which surrounds a smaller centrally located rhabdomere, $\mathrm{R} 7$ in the distal retina (Fig. $2 A$ ) or R8 in the proximal retina (Fig. 2B). Unlike a previous report by Kaminker et al. (2002), however, our findings revealed frequent aberrations ( $>82 \%$ of ommatidia; $N=435$ ) in photoreceptor structure on UAS-runt/+; MT14-GAL4/+ overexpression. In runt overexpression flies (Fig. 2C,D), any number or combination of R1-R6 photoreceptors were aberrant, from one to four per ommatidium. Their rhabdomeres were smaller in diameter and located within the circumference of neighboring R1-R6 rhabdomeres. Rhabdomeres were clearly of one size or another, clearly discriminable and without intermediates. The aberrations are not confined to R2 and R5, the outer photoreceptors in which MT14-GAL4 was previously reported (Kaminker et al., 2002) to drive expression (Fig. $2 C$ ) but are most frequently observed in R1, R3, R4, and R6. Our observations from UAS-mCD8::GFP/+;:MT14-gal4/+ flies indicate that on average four photoreceptors per ommatidium in the larval eye disc have MT14 driver expression. These include prosperoimmunolabeled R7 cells (Fig. 3D) and BOSS-immunolabeled R8 cells (Fig. 3I). Small diameter rhabdomeres are characteristic of R7 and 88 cells, both of which express runt during normal development 


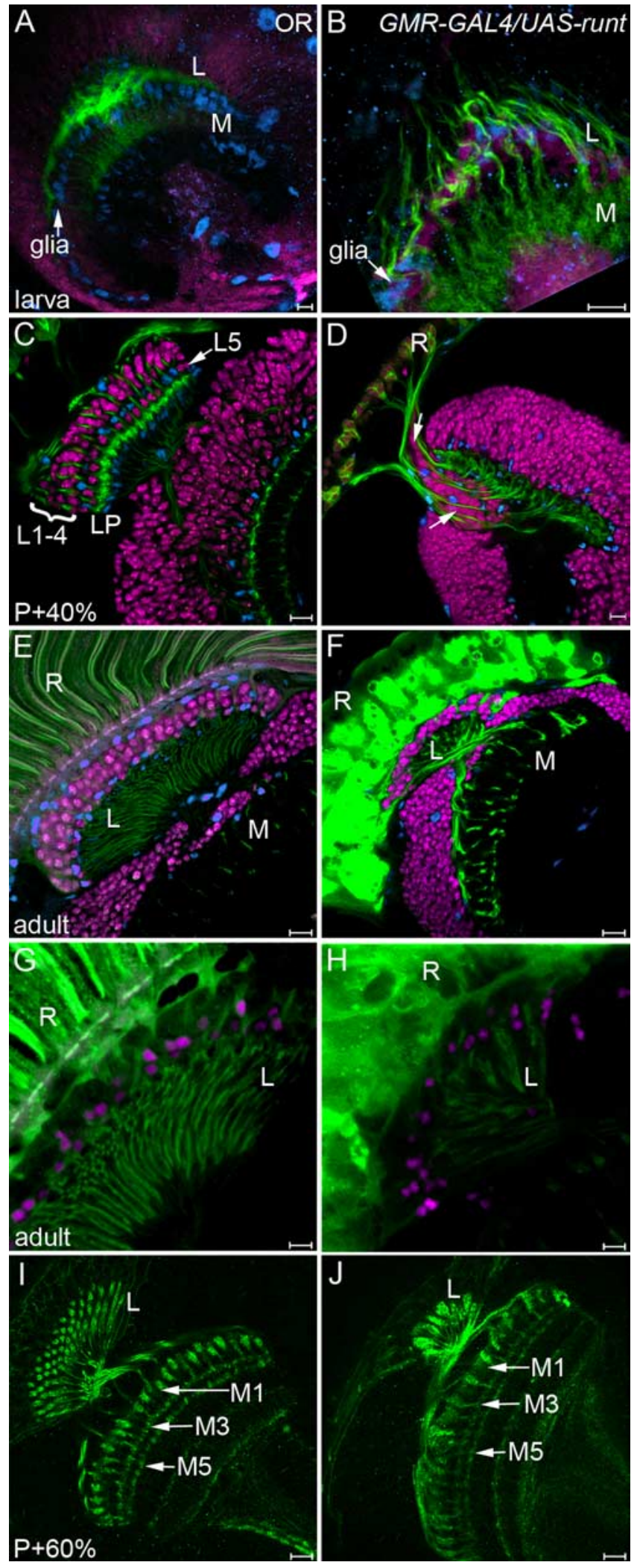

Figure 1. Lamina monopolar cells survive and maintain expression of cell fate markers despite aberrant pathfinding in their photoreceptor inputs. OR (left column) and GMR-GAL4/UAS runt (right column) brains, either third-instar larval whole mounts $(\boldsymbol{A}, \boldsymbol{B})$, or Vibratome slices of pupal $\mathrm{P}+40 \%(\boldsymbol{C}, \boldsymbol{D}), \mathrm{P}+60 \%(\boldsymbol{I}, \boldsymbol{J})$, or adult $(\boldsymbol{E}-\boldsymbol{H})$ brains. $\boldsymbol{A}-\boldsymbol{F}$, Brains are immunolabeled with antibodies against the following markers: photoreceptor-specific Chaoptin (green); Elav (magenta), an RNA-binding protein expressed in postmitotic neurons; and Repo (blue), a nuclear glial antigen. $\boldsymbol{A}$, In 0R larvae, R1-R6 photoreceptors terminate in the lamina (L), between epithelial and marginal glia, whereas R7-R8 terminate in the medulla (M). $\boldsymbol{B}$, When photore-
(Kaminker et al., 2002), leading us to investigate further whether these R1-R6 photoreceptors with smaller rhabdomeres had other alternate photoreceptor characteristics.

To determine whether R1-R6 photoreceptors with transformed rhabdomeres maintained their other characteristic features, we examined Rhodopsin1 (Rh1) driver activity in UASrunt/Rh1-lacZ; MT14-GAL4/+ eyes. Rh1, encoded by the gene ninaE, is the light-absorbing opsin of R1-R6 photoreceptors (O’Tousa et al., 1985) and is expressed late in development after photoreceptor cell fate is fully established. In Rh1-lac Z flies, the $R h 1$ promoter drives expression of exogenous lac $Z$, the protein product of which, $\beta$-gal, is detectable by immunocytochemistry. Rh1 can therefore be used to distinguish R1-R6 from the R7 and R8 photoreceptors, which express different opsins. In the eyes of UAS-runt/Rh1-lacZ; MT14-GAL4/+ flies, we identified three outcomes to this labeling: (1) R1-R6 rhabdomeres are large and all cells express Rh1-driven $\beta$-gal (Fig. $2 E$ ), as in wild type; (2) a small rhabdomere forms in a cell body that has no $\beta$-gal expression (Fig. 2F); or (3) a small rhabdomere forms but its cell body continues to express $R h 1$-driven $\beta$-gal (Fig. $2 G$ ). Of the photoreceptors with smaller rhabdomeres, most $(93 \% ; N=28)$ are from cell bodies that failed to express $\beta$-gal and had thus undergone transformation to an alternative photoreceptor cell fate.

Once these findings had clearly shown that photoreceptor fate was indeed altered, it was imperative to determine whether photoreceptors R1-R6 were transformed to R7 or to R8, for which reason we next sought markers for these specific photoreceptor subtypes. R7 and R8 have different peaks of spectral sensitivity (Hardie and Kirschfeld, 1983). Each photoreceptor type is divided into subclasses depending on the exact Rhodopsin expressed (Morante and Desplan, 2004). There are two subtypes of R8 cell, one that expresses Rh5 (Chou et al., 1996; Papatsenko et al., 1997) and absorbs light with wavelengths in the blue region of the spectrum, and the other that expresses Rh6 and absorbs in the green (Townson et al., 1998; Salcedo et al., 1999). R7 cells also have two subtypes with opsins Rh3 (Fryxell and Meyerowitz, 1987; Zuker et al., 1987) and Rh4 (Montell et al., 1987), both of which absorb light in the UV.

In UAS-runt/+; MT14-GAL4/Rh3-lacZ flies, $\beta$-gal is immunolocalized to a subset of R7 (Fig. $3 A$ ) cells as well as to a subset of small transformed outer rhabdomeres (Fig. $3 B, C$ ). In 37 ommatidia examined, 35 transformed rhabdomeres were identified of which $66 \%$ were immunoreactive for Rh3-driven $\beta$-gal expression, with 33\% expressing an unknown opsin (Fig. 3C). In UASrunt/+; MT14-GAL4/Rh6-lacZ flies, $\beta$-gal is immunolocalized to a small subset of central R8 photoreceptors (Fig. $3 F, G$ ), which in

$\leftarrow$

ceptors overexpress Runt, most axons terminate in the medulla. $\boldsymbol{C}$, In the wild-type pupa, R1-R6 axons terminate at the lamina plexus (LP), above which lie columns of Elav-positive nuclei of lamina neurons (L1-L5). D, Occasionally, the medulla of GMR-GAL4/UAS-runt flies fails to rotate to lie parallel to the retina, as seen here at $P+40 \%$. Elav-expressing nuclei, probably those of L1-L5 (between arrows), lie compressed between en passant photoreceptor axons. In adult GMR-GMR4/UAS-runt flies $(\boldsymbol{F})$, the retina $(\mathrm{R})$ is severely disrupted. $\boldsymbol{E}$, Cell bodies of neurons are located between layers of glia in the wild-type lamina cortex. $\boldsymbol{F}$, Although the lamina of GMR-GAL4/UAS-runt flies is highly condensed, lamina neurons survive into adulthood and are appropriately located beneath the basement membrane of the compound eye. $\boldsymbol{G}, \boldsymbol{H}, \mathbf{B S H}$ immunoreactive nuclei of $L 5$ neurons (magenta) are located just distal to photoreceptor axon terminals (Chaoptin; green) in the lamina of both OR (G) and GMR-GAL4/UAS-runt $(\boldsymbol{H})$ flies. $\boldsymbol{I}, \boldsymbol{J}$, Fasll-immunoreactive L1 and L3 monopolar neurons expand in the lamina as well as in the M1, M5 (L1), and M3 (L3) layers of the medulla, as demonstrated in the wild-type $P+60 \%$ pupa (I). In GMR-GAL4/UAS-runt flies $(\boldsymbol{J})$, $\mathrm{L} 1$ and $\mathrm{L} 3$ continue to express Fasll and extend their axons into the medulla to terminate in the appropriate layers. Scale bars: $\boldsymbol{A}-\boldsymbol{F}, \boldsymbol{I}, \boldsymbol{J}, 10 \mu \mathrm{m} ; \boldsymbol{G}, \boldsymbol{H}, 5 \mu \mathrm{m}$. 
a wild-type ommatidium extend their cell body between R1 and R2. Additional Rh6lac $Z$ expression was associated with small transformed outer rhabdomeres (Fig. 3G). We crossed Rh4-lacZ and Rh5-lacZ into a UAS-runt; MT14-GAL4 background but found expression of neither of these opsins in transformed outer rhabdomeres (data not shown). LacZ expression was virtually eliminated in UAS-runt; MT14-GAL4/ Rh5-lacZ eyes (data not shown). This loss was unexpected considering that many central R8 photoreceptors in UAS-runt/+; MT14-GAL4/Rh6-lacZ do not have Rh6lac $Z$ expression (Fig. $3 H$ ). It is unknown what opsin these R8 photoreceptors express.

Despite the presence of excess R7 and R8 photoreceptors in the adult, Prospero and BOSS immunolabeling of larval eye discs did not reveal excess $\mathrm{R} 7$ (Fig. $3 D, E$ ) or R8 (Fig. $3 I, J$ ) cells in UAS-mCD8::GFP/+;UAS-runt/+; MT14GAL4/+ flies (Fig. $3 E, J$ ) when compared with the wild-type UAS-mCD8::GFP/+;; MT14-GAL4/+ (Fig. 3D,I) eye disc, similar to the findings of Kaminker et al. (2002). The lack of excess R8 photoreceptors in the eye disc is not surprising given that BOSS expression precedes MT14GAL4-driven UAS-mCD8::GFP expression in the larval eye disc (data not shown), and thus would also precede runt overexpression using this driver line.

\section{Effects of Runt overexpression on R7 and R8 photoreceptor terminals}

R7 and R8, the two central photoreceptors of the ommatidium, have axons that terminate in two distinct layers of the medulla, R8 in the distal stratum M3, and R7 deeper, in stratum M6 (Fischbach and Dittrich, 1989). There, processing of the different spectral inputs from each type of terminal is presumed to occur in stratumspecific circuits (Morante and Desplan, 2004). We initially used photoreceptor-

specific Chaoptin immunolabeling to visualize terminals in the medulla, making it impossible to determine the individual contributions from R7, R8, or ectopic R1-R6 photoreceptor terminals, which thus required us to use photoreceptor subtypespecific markers.

Before attempting EM analysis of the medulla, we examined the structure of R7 and R8 terminals in mutant flies at the light microscope level. R8 terminals were visualized using $R h 5$-lac $Z$ and Rh6-lacZ, in GMR-GAL4,UAS-runt/+ and UAS-runt; MT14-GAL4 (data not shown) flies. These lacZ lines clearly labeled distinct subsets of wild-type R8, both of which are swollen in two locations in the medulla, distally, just above M1, and at their enlarged terminals in stratum M3, as shown here for Rh6lacZ (Fig. 4A,B). GMR-GAL4-driven overexpression of runt (Fig. $4 C, D)$ resulted in more slender $R h 6$-lacZ-expressing $\mathrm{R} 8$ terminals, some of which bypassed M3 to terminate deeper at M6 (Fig.
4D). Rh6-expressing R8 terminals have a disorganized appearance in GMR-GAL4,UAS-runt (Fig. 4C) medullas when compared with wild-type (Fig. $4 A$ ) terminals visualized in a $\sim 40 \mu \mathrm{m}$ depth of tissue. In GMR-GAL4,UAS-runt/CyO; Rh5-lacZ/+ flies, as was previously noted in the eye, $R h 5$-driven $\beta$-gal expression is almost completely lost, with only two R8 terminals detected in the 12 brains that were analyzed (data not shown).

To examine R7 terminals after runt overexpression, the Rh3lacZ and Rh4-lacZ lines were used. As seen in Rh3-lacZexpressing flies, wild-type R7 axons expanded in the M6 layer of the medulla where they terminated (Fig. $4 F$ ), behavior that was replicated in the Runt overexpression flies (Fig. $4 H$ ), yet not all R7 axons were successful in extending and maintaining terminals down into the M6 layer of the adult medulla. Furthermore, in runt overexpression flies, R7 terminals were more numerous (Fig. 4G) than in the wild type (Fig. 4E) but do not appear to 

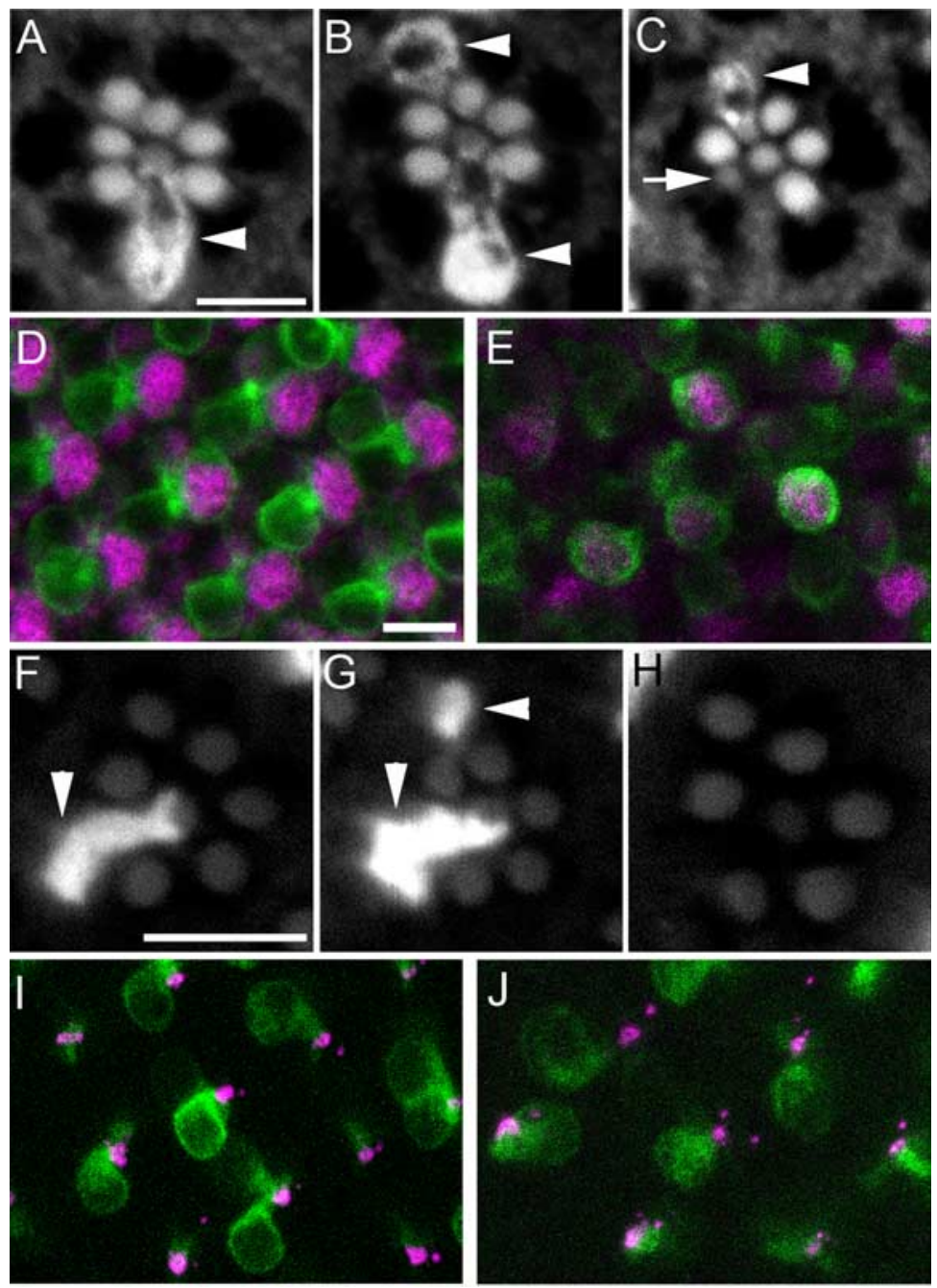

Figure 3. Transformed R1-R6 photoreceptors express Rh3 and Rh6. In UAS-runt/+;MT14-GAL4/Rh3-lacZ flies (A-C), $\beta$-gal expression is localized to R7 photoreceptors. In a wild-type ommatidium, $\beta$-gal expression was limited to the cell body of the R7 photoreceptor, which has a small centrally located rhabdomere $(\boldsymbol{A})$. In ommatidia with an excess of small rhabdomeres, $\beta$-gal is immunolocalized to a subset of cells with small rhabdomeres, seen here in at the R3 position (arrowheads), independent of whether or not the central R7 also expressed $\beta$-gal $(\boldsymbol{B}, \boldsymbol{C})$. Not all transformed photoreceptors expressed Rh3-driven $\beta$-gal expression (C, arrow). UAS-runt/+;MT14-GAL4/Rh6-lacZflies $(\boldsymbol{F}-\boldsymbol{H})$ expressed $\beta$-gal in some R8 photoreceptors. In a wild-type ommatidium, the R8 rhabdomere is centrally located and the cell body protrudes between R1 and R2 ( $\boldsymbol{F}$, arrowhead). However, in some mutant ommatidia, outer photoreceptors, such as the one in the R3 position (G, arrowhead), are converted to Rh6expressing R8 cells. Despite an almost complete lack of Rh5 expression (data not shown), many ommatidia have a central R8 photoreceptor with no Rh6 expression $(\boldsymbol{H})$. MT14-GAL4 drives UAS-mCD8::GFP expression (green) in R7 cells (Prospero; magenta; $\boldsymbol{D}, \boldsymbol{E}$ ), and R8 cells (BOSS; magenta; $\boldsymbol{I}, \boldsymbol{J}$ ); however, no excess of Prospero-expressing R7 nuclei $(\boldsymbol{E})$ or B0SS-expressing R8 nuclei $(\boldsymbol{J})$ are detected in the larval eye disc of UAS-mCD8::GFP;UAS-runt;MT14-GAL4 flies (E) when compared with the wild-type eye discs $(\boldsymbol{D}, \boldsymbol{I})$. Scale bars: (in $\boldsymbol{A}) \boldsymbol{A}-\boldsymbol{C}$; (in $\boldsymbol{D}) \boldsymbol{D}, \boldsymbol{E}, \boldsymbol{I}, \boldsymbol{J}$; (in $\boldsymbol{F}) \boldsymbol{F}-\boldsymbol{H}, 5 \mu \mathrm{m}$.

cluster into the "blebs" characteristic of Rh1-lacZ-expressing terminals in the medulla of mutant flies (Fig. 5B). In Rh4-lacZ/GMRGAL4,UAS-runt flies, there is a reduction in Rh4-lacZ-expressing terminals, which would normally constitute $70 \%$ of wild-type R7 cells (Franceschini et al., 1981). Rh4-lacZ expression was limited to the anterior region of the eye and thus to terminals in the posterior medulla when a strong GAL4 driver is used, such as in Rh4-lacZ/ GMR-GAL4, UAS-runt, but was more widely expressed in Rh4/lacZ/ UAS-runt; MT14-GAL4/+ flies (data not shown).

\section{Medulla terminals of mistargeted R1-R6 photoreceptors express synapse-associated proteins}

To recognize ectopic terminals in the adult medulla, we used the Rh1-lacZ expression construct in flies with both UAS-runt and
MT14-GAL4, in a similar strategy as that for R7 and R8, above. In the phenotypically wild-type lamina (Fig. 5A), R1-R6 axons converge on a cartridge and each swells to form a cylindrical synaptic terminal running the depth of the lamina. In UAS-runt/Rh1-lacZ; MT14-GAL4/+ flies, some R1-R6 photoreceptors maintain the appropriate Rh1 driver expression yet terminate ectopically in the medulla. When the axons from R1-R6 extend to the medulla their terminals fail to form elongated cylindrical structures, as they do in the lamina. Instead, swelling is intermittent, occurring at different levels within the medulla so as to form blebs along the length of the axons (Fig. 5B). Axons extended into the medulla in bundles, but we were not able to discern whether these blebs represented multiple swellings along the length of a single axon or the terminal swellings of different photoreceptors in a single axon bundle, each of which terminated at a different stratum in the medulla. Moreover, these axonal swellings were not restricted to the M3 and M6 layers, the normal terminal locations of the R8 and R7 photoreceptors.

To determine whether the blebs were sites of synaptic specializations, we used antibodies against known synaptic or synapse-associated proteins. Photoreceptor synaptic zones have distinct specializations that include many synaptic vesicles, capitate projections, and synaptic T-bar ribbons that comprise a pedestal, anchoring proteins and a surmounting platform (Prokop and Meinertzhagen, 2006). Synaptic vesicles were recognized by their association with CSP (Zinsmaier et al., 1990), which is involved in $\mathrm{Ca}^{2+}$. dependent exocytosis (Zinsmaier et al., 1990; Chamberlain and Burgoyne, 2000; Evans et al., 2003). CSP immunolabeling is localized to all areas of synaptic release, including the R1-R6 photoreceptor terminals in the lamina of wild-type flies (Fig. 5C). In runt overexpression mutants, CSP continued to be expressed in the lamina and medulla (Fig. $5 D$ ), and overlapped $R h 1$-driven $\beta$-gal around the profile perimeters of photoreceptors in the lamina (Fig. 5G) and ectopic photoreceptor terminal blebs in the medulla (Fig. $5 H$ ). The overlap suggests that these blebs contained synaptic vesicles and were thus candidate sites for synapses. Presynaptic sites also contain Bruchpilot, a coiled-coil domain protein localized to the active zone of neuromuscular junctions and the optic neuropiles (Fig. $5 E$ ) by the antibody nc82 (Kittel et al., 2006). In UAS-runt/Rh1-lacZ; MT14-GAL4/+ flies, nc82 colocalized with $\beta$-gal expression (Fig. $5 F$ ) to photoreceptors in the lamina (Fig. $5 I$ ) and to terminals of ectopic R1-R6 photoreceptors in the medulla (Fig. 5J). This colocalization suggests that these terminals contained the T-bar ribbons at which synaptic release occurs.

We wanted to determine whether an individual Runt- 

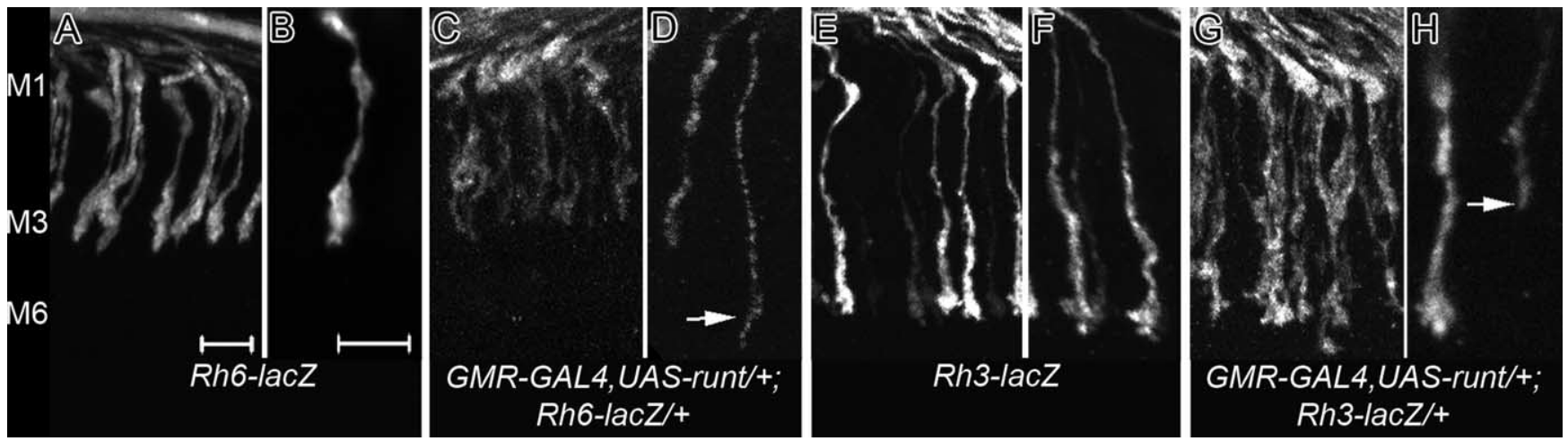

Figure 4. Runt overexpression affects the terminals of photoreceptors R7 and R8 in the medulla. Terminals of R7 and R8 visualized by $\beta$-gal immunolabeling from their expression of Rh3-lacZ (R7; $\boldsymbol{E}-\boldsymbol{H}$ ) or Rh6-lacZ (R8; $\boldsymbol{A}-\boldsymbol{D})$. For $\boldsymbol{A}, \boldsymbol{C}, \boldsymbol{E}, \mathbf{G}$, images of axon terminals were captured from a $41 \mu \mathrm{m}$ depth of tissue. In the wild-type medulla, all Rh6-lacZ-expressing axons terminate in the M3 layer of the medulla $(\boldsymbol{A})$ and have synaptic expansions at their terminal ends $(\boldsymbol{B})$. After ectopic Runt expression in GMR-GAL4, UAS-runt/+; Rh6-lacZ/+ R8 axons in the medulla are less organized ( $\boldsymbol{C}$. There did not appear to be a change in the overall number of axons $(\boldsymbol{C})$; however, terminals appeared narrower and some extended erroneously into M6 (D, arrow). $\boldsymbol{E}-\boldsymbol{H}$, Rh3-lacZ-expressing axons terminate and expand in the M6 layer of the medulla $(\boldsymbol{E}, \boldsymbol{F}), \boldsymbol{G}, \boldsymbol{H}$, After ectopic Runt expression, there are many more Rh3-lacZ-expressing R7 terminals $(\boldsymbol{G})$, and although the shape of correctly targeting terminals is maintained, some R7 axons terminate short of $M 6$ ( $H$, arrow). Scale bars: (in $\boldsymbol{A}) \boldsymbol{A}, \boldsymbol{C}, \boldsymbol{E}, \boldsymbol{G}$; (in $\boldsymbol{B}) \boldsymbol{B}, \boldsymbol{D}, \boldsymbol{F}, \boldsymbol{H}, 5 \mu \mathrm{m}$.

overexpressing photoreceptor was capable of swelling and expressing synaptic proteins in both the lamina and medulla. To do this, we used a MARCM-style approach to label individual photoreceptors, which expressed both UAS-runt and $U A S-m C D 8:: G F P$ under control of the GMR-GAL4 driver. Horizontal slices of adult $h s F L P / U A S-m C D 8:: G F P$; NeoFRT40A actin-GAL80/NeoFRT40A, GMR-GAL4, UAS-runt fly heads colabeled with antibodies against GFP and Bruchpilot (nc82) revealed that most GFP-expressing photoreceptor axons expanded and terminated either in the lamina (Fig. $5 L, N$ ), or alternatively bypassed the lamina (Fig. $5 M$ ) to expand and terminate in the medulla. Furthermore, the photoreceptor phenotypes that characterize cell fate transformation after Runt overexpression using either GMR-GAL4 (Fig. $1 F$ ) or MT14-GAL4 (Fig. 2) drivers, were not observed in GFP-expressing cells in the MARCM eye. So, R1-R6 MARCM photoreceptors expressing GFP, and thus also overexpressing Runt under control of GMRGAL4, had large rhabdomeres characteristic of wild-type R1-R6 (Fig. $5 K$ ).

Many Runt, GFP-overexpressing cells were found in the retina. Of all ommatidia $(N=302), 53 \%$ contained either an R7 or R8 cell expressing GFP. Some of these could also contain R8 or R7, but because they are tiered these could not be seen in a single section; most sections were cut at the R7 level. Twenty-four percent of all R1-R6 cells $(N=1830)$ were labeled. These relatively large numbers of cells made it difficult to discern the axons of individual cells, isolated from those of their neighbors.

Some axons (less than two per brain) appeared to expand in both the lamina and medulla (Fig. 5O,P). Clear images of these required considerable searching. Unlike wild-type R7 or R8 axons, which do not expand in the lamina (Fig. 5I), these axons were enlarged in both the lamina (Fig. $5 O^{\prime}, P^{\prime}$ ) and medulla, shown terminating in the distal medulla near M3 (Fig. 5O' $P^{\prime \prime}$ ). These axons were wider along their entire length than those of wild-type R7 or R8 cells, and also had atypical expansions in the chiasm (Fig. 5O). It is not known whether these are single or multiple, bundled axons; the fact that the labeled profile terminated in only a single medulla stratum (M3) suggested that it was not the bundled axons of both R7 and R8. Furthermore, because R1-R6 rhabdomeres were not transformed to have smaller rhabdomeres like those of R7-R8, suggested that any individual ommatidium does not project a bundled pair of R8 axons to the medulla that terminates in M3. Together, these considerations suggest that the occasional large axons may be from single photoreceptors: either an R8 cell that expands abnormally in the lamina or an R1-R6 cell that terminates ectopically in the medulla. The phenotype after MARCM-style Runt overexpression is considerably less severe than that observed after Runt overexpression alone, because it lacks photoreceptor cell fate transformations and has fewer axon termination errors.

\section{R1-R6 photoreceptors continue to form synapses in the lamina}

To interpret the lamina phenotype that results when Runt is expressed in R1-R6 first requires explanation of the normal axon trajectories of R1-R6 in the adult lamina. In the wild-type lamina, axon bundles from each ommatidium innervate the lamina cortex, and the six photoreceptor axons from R1-R6 then diverge from their bundle and sort into different cartridges (TrujilloCenóz, 1965; Braitenberg, 1967), according to the principle of neuronal superposition (Braitenberg, 1967; Kirschfeld, 1967). Each lamina cartridge is thus a module comprising these six R1-R6 terminals and the fixed group of lamina cells they innervate (Fig. $6 \mathrm{~A}$ ). Along the axis of the cartridge the axons of two lamina cells, $\mathrm{L} 1$ and $\mathrm{L} 2$, extend dendrites that embrace the terminals of R1-R6 and with other lamina cells form tetrad synapses (Fig. 6C-F) (Meinertzhagen and O'Neil, 1991). These are sites of release of the photoreceptor neurotransmitter, histamine (Hardie, 1987). Unlike R1-R6, the axons of R7 and R8 extend alongside the cartridge without synaptic engagement.

Flies with exogenous Runt expression in R1-R6 have disordered lamina cartridges but still display features characteristic of wild-type photoreceptor terminals. The laminas of both GMRGAL4 (data not shown) and MT14-GAL4-driven UAS-runt flies are highly disorganized (Fig. $6 B$ ). Photoreceptor profiles bundle with the axons of lamina neurons and are surrounded by epithelial glia to form a disordered association. Such aberrant cartridges contain the expanded synaptic profiles typical of R1-R6 terminals but are formed by axons that in fact may neither sort into cartridges nor even terminate in the lamina. Other axon profiles are possibly R1-R6 axons that, like the normal profiles of R7 and $\mathrm{R} 8$, also bypass the lamina without forming synapses at that particular level. They may also be axons from R1-R6 cells that have transformed into supernumerary R7 and R8 cells. Expanded pho- 

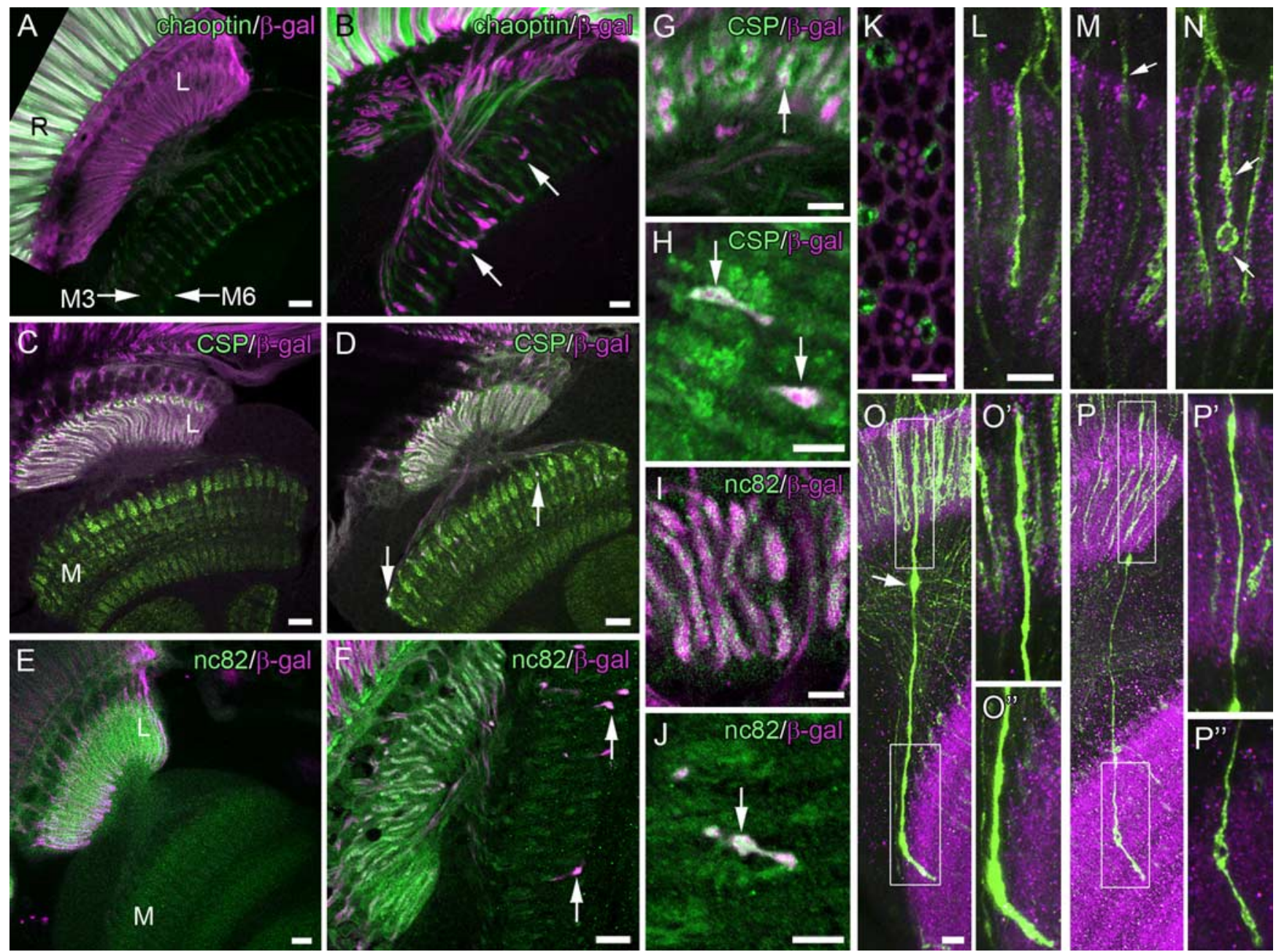

Figure 5. Ectopic R1-R6 photoreceptors in the medulla (M) visualized with Rh1-lacZ.A, B, Photoreceptors express Rh1-lacZ (R1-R6: $\beta$-gal, magenta) and anti-chaoptin immunolabeling (R1-R8, green). $A, C, E, R 1-R 6$ photoreceptors terminate exclusively in the lamina (L) of $R h 1$-lacZ flies. $B, D, F$, In Rh1-lacZ/UAS-runt; MT14-GAL4/+ flies, ectopic R1-R6 axons innervate the medulla where they extend as deep as stratum $\mathbf{M 6}$, forming bleb-like swellings along their length ( $\boldsymbol{B}$, arrows). $\boldsymbol{C}, \boldsymbol{D}, \boldsymbol{G}, \boldsymbol{H}$, Areas expressing the vesicle-associated protein, CSP, are candidate sites of synaptic release visualized in single image planes $(0.8 \mu \mathrm{m})$ from a Vibratome slice. C, CSP (green) colocalized (white) to $\beta$-gal immunoreactivity (magenta) of R1-R6 photoreceptor terminals in the wild-type lamina. $\boldsymbol{D}, \boldsymbol{G}, \boldsymbol{H}$, CSP also colocalized (arrows) to $\beta$-gal around the perimeter of R1-R6 photoreceptor terminals in the mutant lamina $(\boldsymbol{D}, \boldsymbol{G})$ and ectopic terminals in the medulla $(\boldsymbol{D}, \boldsymbol{H}) . \boldsymbol{E}, \boldsymbol{F}, \boldsymbol{I}, \boldsymbol{J}, \mathrm{The}$ T-bar ribbon-associated protein Bruchpilot is immunolocalized to presynaptic sites with the antibody nc82 (green), and was detected in the lamina of both $R h 1$-lacZ flies (E) and mutant $(\boldsymbol{F}, \boldsymbol{I})$ flies, as well as in ectopic R1-R6 terminals (magenta, white) in the medulla $(\boldsymbol{F}, \boldsymbol{J}$, arrow). In the hsFLP/UAS-mCD8::GFP; NeoFRT40A actin-GAL80/NeoFRT40A, GMR-GAL4, UAS-runt (MARCM) visual system $(\boldsymbol{K}-\boldsymbol{P})$ subsets of Runt overexpressing photoreceptors (green cells in $\boldsymbol{K}$ ) and their axons (projection images from confocal stacks in $\boldsymbol{L}-\boldsymbol{P}$ ) are labeled by mCD8::GFP (green), and the neuropils are colabeled with nc82 (magenta). In the lamina $(\boldsymbol{L}-\boldsymbol{N})$, most photoreceptor axons have a structure resembling that of wild-type R1-R6 terminals $(\boldsymbol{L})$, are through-going like wild-type R7-R8 axons $(\boldsymbol{M}$, arrow), or have an abnormal terminal with varicosities (arrows) along its length $(\boldsymbol{N})$. Occasional photoreceptor axons appear to form synaptic swellings in both optic neuropils $(\boldsymbol{O}, \boldsymbol{P})$. These axons expand in both the lamina (enlarged in $\left.\mathbf{O}^{\prime}, \boldsymbol{P}^{\prime}\right)$ and medulla (enlarged in $\left.\mathbf{O}^{\prime \prime}, \boldsymbol{P}^{\prime \prime}\right)$, and occasionally along the axon during its passage in the chiasma $(\boldsymbol{O}$, arrow). Scale bars: $\boldsymbol{A}-\boldsymbol{F}, 10 \mu \mathrm{m} ; \boldsymbol{G}-\boldsymbol{K}, 5 \mu \mathrm{m}$; (in $L$ ) $L, M, N, 5 \mu \mathrm{m}$; (in 0$) \mathbf{O}, P, 5 \mu \mathrm{m}$.

toreceptor profiles in these aberrant cartridges resemble wildtype R1-R6 terminals in containing capitate projections (Trujillo-Cenóz, 1965), synaptic vesicles, mitochondria, and tetrad synapses (Fig. 6G-I). Capitate projections are photoreceptor-specific organelles, sites of endocytotic recovery of synaptic vesicle membrane and also proposed sites for localized histamine recycling (Fabian-Fine et al., 2003). The presence of this suite of organelles suggests that these terminals possess the means for synaptic release, whereas the presence of mitochondria suggests that they are energetically equipped to do so (GórskaAndrzejak et al., 2003).

Our findings from mutant lamina ultrastructure also reveal that in UAS-runt/+; MT4-GAL4/+ flies, the axons of R1-R6 photoreceptors continued to form reciprocal synaptic inputs with lamina neurons, most probably with their normal targets in that neuropile. Feedback synapses onto R1-R6 are found in the distal lamina, although we have not identified the profiles presynaptic to these mutant photoreceptors (Fig. $6 J$ ). In the wild type, most synaptic feedback comes from amacrine cells, with fewer contributions from L2 and L4 in the distal lamina (Meinertzhagen and O’Neil, 1991).

Supernumerary photoreceptors form synapses in the medulla When GMR-GAL4 is crossed into the UAS-HRP::CD2 reporter construct, the axons of all photoreceptor neurons can be identified in electron micrographs. In UAS-HRP::CD2-expressing flies, HRP is localized to the membranes of cells and can be visualized in EM from the formation of an electron-dense precipitate after incubation with $\mathrm{DAB}$ and $\mathrm{H}_{2} \mathrm{O}_{2}$ (Larsen et al., 2003). In the wildtype Drosophila lamina, the slender axons of R7 and R8 extend alongside the cartridge of their retinal R1-R6 neighbors (Fig. 7A). They penetrate the lamina and innervate the medulla, where in 
the distal strata, beneath M1, their profiles were normally small and unexpanded (Fig. $7 B$ ). Exposure to $\mathrm{DAB} / \mathrm{H}_{2} \mathrm{O}_{2}$ revealed large terminals with electron-dense membrane in the distal medulla of both GMR-GAL4/ UAS-runt; UAS-HRP::CD2/+ (data not shown) and UAS-HRP::CD2/UAS-runt; MT14-GAL4/+ flies (Fig. 7C). These photoreceptor axons tended to cluster in groups and form terminal swellings (Fig. 7D). Counts of photoreceptor terminal profiles from the medullas of two flies indicate variation in the number of photoreceptors terminals per column. The number of terminals per cluster ranged from 3 to 8 , with an average of 4.77 terminals per column $(N=13$ columns $)$, more than the normal 2 profiles (R7 and R8).

Supernumerary photoreceptor terminals in the medulla contained many of the features characteristic of wild-type R1-R6 photoreceptor terminals in the lamina. These included the mitochondria, vesicles, presynaptic T-bar ribbons and, most notably, capitate projections. The hyperinnervation of the medulla that results from supernumerary R7 photoreceptors and ectopically projecting R1-R6 photoreceptor axons appeared to be fully supported by the medulla target cells, which imposed no clear restriction to the formation of novel photoreceptor synapses. We analyzed nine columns with supernumerary photoreceptor clusters through a depth of 120-360 nm. Of these, at least eight columns had synapses in three distinct photoreceptor terminals, indicating that a column can support more photoreceptors than those two terminals (R7, R8) normally present in a wild-type medulla column. Furthermore, in four of nine columns, $100 \%$ of the supernumerary terminals contained synapses, sometimes with up to eight photoreceptor terminals forming input synapses to a column.

Although supernumerary photoreceptors in the medulla were able to form synapses complete with a T-bar ribbon, what was perhaps most striking was the number of postsynaptic partners at some release sites. Tetrads were readily detected in the medulla (Fig. $7 J-M$ ), just as R1-R6 would normally form in the lamina. The normal synapses of wild-type R7 (Fig. 7E-I) and R8 terminals, in contrast, can form tetrads but are mostly triads, with three postsynaptic partners (Takemura et al., 2008). For synapse counts in supernumerary terminals, a 350 $\mathrm{nm}$ depth of tissue was examined for seven columns each of which contained more than four photoreceptor terminals. We identified 35 synapses, 22 of which were tetrads. Of synapses that could be traced through their depth, 2 of 25 were triads, and 1 of 25 was a dyad. Of those which could not be traced entirely through their depth, 9 of 10 were at least triads and the remaining synapse was at least a dyad. These numbers probably reflect incomplete tracing rather than incomplete tetrads, and their pro-

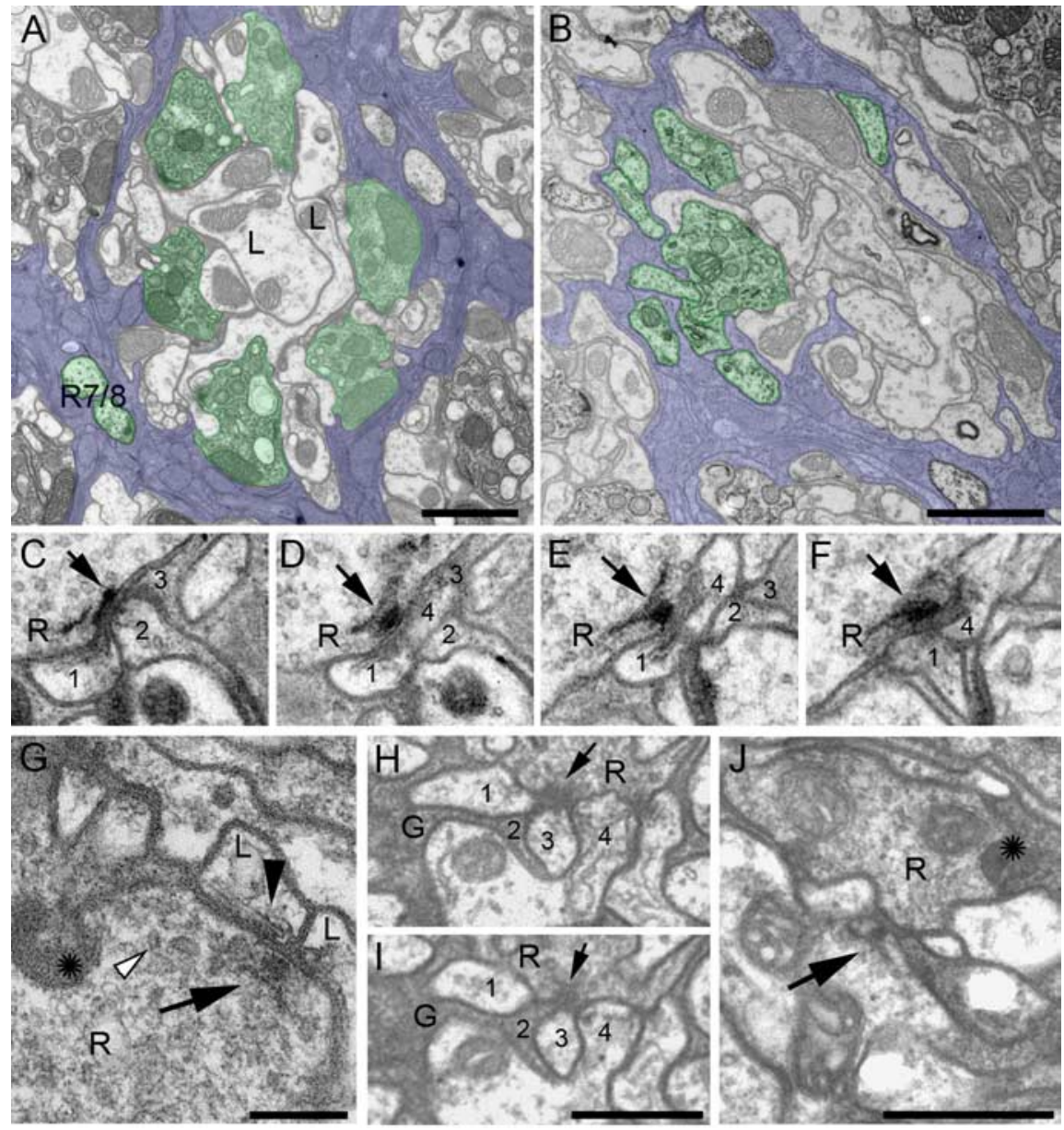

Figure 6. Flies with exogenous Runt expression in R1-R6 have disorganized lamina cartridges, but the axons of R1-R6 have terminals with features characteristic of those in the wild type. $\boldsymbol{A}$, Wild-type organization of a lamina cartridge in UAS-HRP::CD2/+;MT14-GAL4/+ flies. A cartridge contains photoreceptor terminals (green) that originate from R1-R6 in neighwhaptic organelles, and also the unexpanded profiles of en passant photoreceptor axons. $\boldsymbol{C}-\boldsymbol{F}$, Consecutive EM sections of 1-4). G-J, Photoreceptors in UAS-runt/+; MT14-GAL4/+ flies resemble wild-type terminals of R1-R6 by having capitate Consecutive $60 \mathrm{~nm}$ sections through a synapse. Mutant synapses have four postsynaptic partners (labeled 1, 2, 3, 4) and thus formally are tetrads. $J$, Forming possible feedback synapses (arrow), other neurons are presynaptic to photoreceptors. Scale bars: A, B, $1 \mu \mathrm{m} ; \boldsymbol{G}, 0.2 \mu \mathrm{m} ; \boldsymbol{I}, \boldsymbol{J}, 0.5 \mu \mathrm{m}$.

portions compare with those found in R1-R6 in the wild-type lamina [unpublished analyses of data reported by Meinertzhagen and Sorra (2001)]. Numerical conservation of the postsynaptic ensemble suggests that this tetrad organization is determined cell autonomously by the R1-R6 photoreceptors. In the lamina, the tetrad incorporates a blend of postsynaptic elements from lamina neurons L1, L2, L3, amacrine cells, and epithelial glia (Meinertzhagen and O'Neil, 1991). Although the postsynaptic partners at the ectopic R1-R6 are not known, the distal medulla does contain axon terminals from the normal lamina constituents of the tetrad, L1-L3 (Fischbach and Dittrich, 1989). It is therefore possible that R1-R6 may synapse in the medulla as they would do in the lamina, with any combination of these three cells, but without the usual lamina amacrine and epithelial glial cells, and 

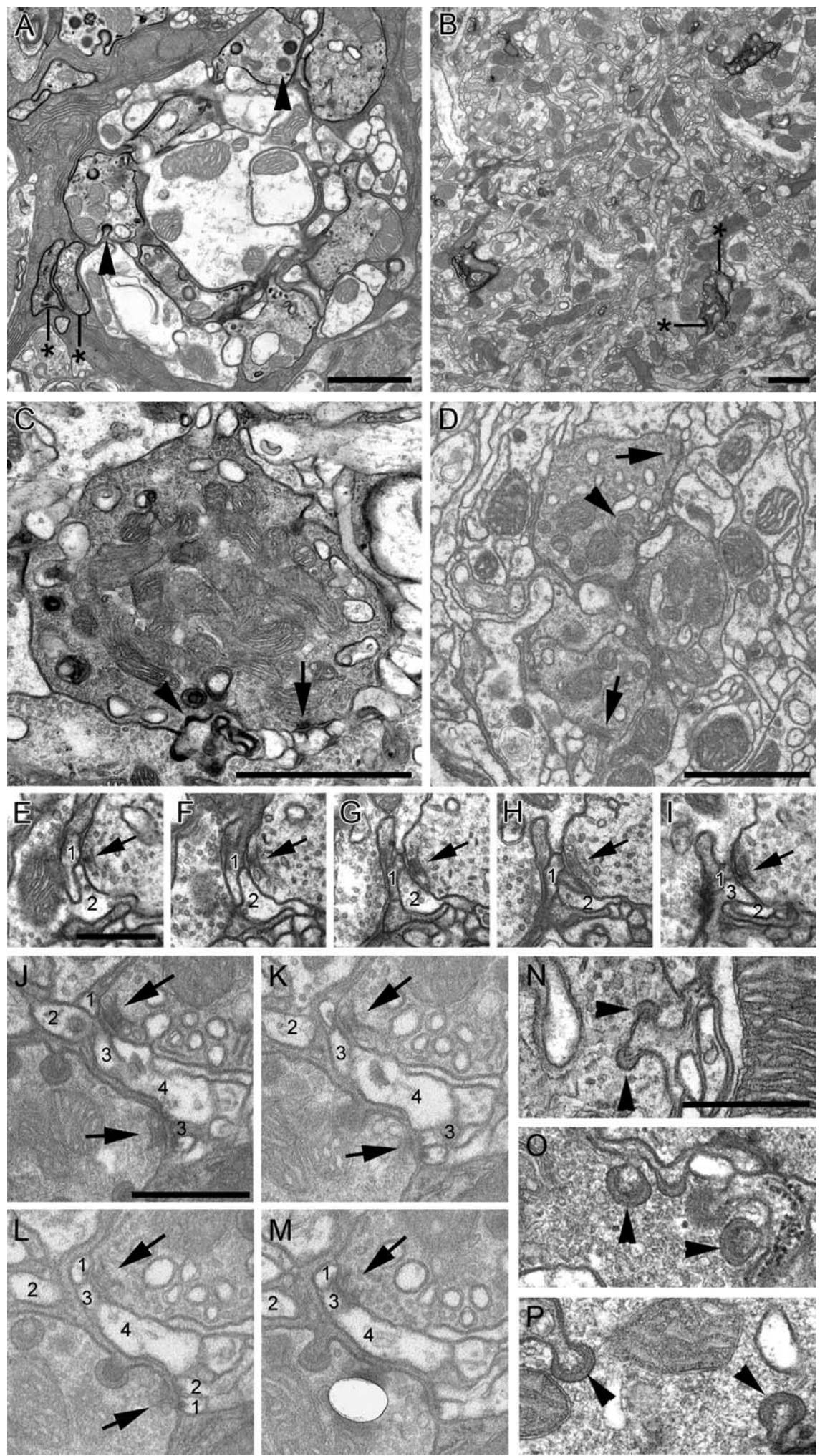

Figure 7. Profiles of photoreceptors in the medulla can be distinguished by the presence of electron-dense DAB on their membranes. $\boldsymbol{A}, \boldsymbol{B}$, Fifty-nanometer cross sections of DAB-labeled GMR-GAL4/UAS-HRP::CD2 brains. $\boldsymbol{A}$, A wild-type lamina cartridge in which the membranes of all photoreceptors, including the penetrating axons of R7 and R8 (asterisks), were labeled with $D A B$. Visible in terminals of R1-R6 are heads of capitate projections (arrowheads). $\boldsymbol{B}$, Unexpanded paired axons of R7/R8 (asterisks) in three columns of the wild-type distal medulla. $\mathbf{C}-\boldsymbol{H}$, Sections through DAB-labeled UAS-runt/UAS-HRP::CD2; MT14GAL4/+ flies. C, Ectopic photoreceptors in the distal medulla can be distinguished with DAB labeling, and because they have capitate projections (arrowhead) in their terminals (compare $\boldsymbol{A}$ ). These terminals also contain presynaptic sites (arrow). $\boldsymbol{D}$ Photoreceptors are distinguished even in the absence of DAB labeling, because they have capitate projections (arrowhead) and because transformed R1-R6 frequently form clusters of cylindrically shaped axons, such as this trio, not found in wild-type medullas. E-I, Series of micrographs showing the triad synapse (arrow) of a wild-type R7 photoreceptor and its three postsynaptic only if such synapses form distal to stratum M2, where the axon of monopolar cell L2 normally terminates. Given that the second expansion of the terminal of L1 lies in stratum M5 of the medulla, whereas ectopic R1-R6 photoreceptors extend as deep as M6 (Fig. 5B), any synapses formed by ectopic R1-R6 photoreceptor terminals in stratum M6 must likewise be formed with novel partners. We conclude that many synapses formed by ectopic R1-R6 terminals provide inputs to at least some novel target neurons that are of medulla origin.

Even in the absence of DAB labeling in the medulla, supernumerary photoreceptor terminals were obvious, revealed by the presence of capitate projections. These had a spherical head, the shape and size (175 \pm $27 \mathrm{~nm}$ ) (Fig. 7O) of which did not differ significantly $(p=0.49$ in a two-tailed $t$ test) from their counterparts in lamina terminals of R1-R6 (192 $\pm 20 \mathrm{~nm})($ Fig. $7 P)$. Those of photoreceptor terminals in the lamina or medulla of UAS-runt/+; MT14GAL4/+ flies likewise did not differ significantly in size ( $p=0.34$ for the medulla; $p=0.99$ for the lamina) from capitate projections in the wild-type lamina (194 \pm 15 $\mathrm{nm}$ ). Wild-type R7 and R8 terminals also received invaginations that resemble capitate projections, and that arose from invaginating medulla glia $(95.4 \pm 11.8 \mathrm{~nm})$ (Fig. $7 N$ ), but their size and shape in R7 and R8 photoreceptors differed from those at both the mutant lamina and medulla sites. Thus, photoreceptors revealed a conserved synaptic architecture among their capitate projections in the medulla, even in the absence of the epithelial glia of the lamina, and were invaginated instead by surrounding medulla profiles that resembled glia, although lamina and medulla have genetically distinct subsets of glia (Tix et al., 1997). The invaginating glia may have been the medulla neuropile glia

elemnts labeled 1-3.J-M, Series of micrographs confirming that ectopic photoreceptors form tetrad synapses (arrows). The unidentified postsynaptic partners for two neighboring synapses (arrows) are labeled 1-4. Element 4 is postsynaptic at both synapses. $\boldsymbol{N}-\boldsymbol{P}$, The shape and size of capitate projections differs in R1-R6 from their counterparts in R7-R8 terminals. Cross-sectioned heads of mushroom-shaped wildtype (OR) R8 capitate projections $(\boldsymbol{N})$ differ in shape and are smaller in diameter than heads of capitate projections from R1-R6 photoreceptors in either the lamina $(\boldsymbol{P})$ or in ectopic photoreceptors in the medulla $(\mathbf{0})$ of UAS-runt/+; MT14GAL4 flies. Capitate projections of mutant R1-R6 in both the lamina and medulla are similar in size to those in wild-type lamina terminals (compare $\boldsymbol{A}$ ). Scale bars: $\boldsymbol{A}-\boldsymbol{C}, 1 \mu \mathrm{m} ; \boldsymbol{D}, 2$ $\mu \mathrm{m} ; \boldsymbol{E}-\boldsymbol{P}, 0.5 \mu \mathrm{m}$. 
that express ebony and are presumed to regulate the metabolism of normal medulla histamine released from R7 and R8 (Richardt et al., 2002). From the size similarity of R1-R6 capitate projection heads in the two locations, and the difference between capitate projections in wild-type $\mathrm{R} 7$ and $\mathrm{R} 8$, we conclude that capitate projection head size is independent of the subtype of glial cell that invaginates the photoreceptor terminal. Rather, the similarity strongly suggests that this feature of organelle architecture is determined by the common element in both, the photoreceptor terminal.

\section{Discussion}

Our data support three main findings: first, that when some of the R1-R6 photoreceptors overexpress runt, either these cells or their neighbors can adopt an alternative fate; second, that when genetically misdirected to a foreign neuropile, one which mostly comprises medulla interneurons that are novel targets, Rh1expressing R1-R6 nevertheless form synapses; and, third, that the presynaptic terminal determines the architecture of its synaptic organelles, including capitate projections, without reference to target neurons.

\section{R1-R6 photoreceptors that overexpress runt often adopt alternative fates}

Developing neurons in the brain normally undergo a sequence of interactions with their neighbors that ensures that each neuron acquires a distinct suite of phenotypic features: axon targeting, synaptic partnerships, etc. These features constitute the fate of that neuron in the brain. Overexpressing runt in R1-R6 transforms the suite of features that normally distinguishes these photoreceptors from their neighbors R7 or R8. We define the cells from the positions they adopt in the ommatidium, which are inherited from the pattern of recruitment of cells during ommatidial assembly (Tomlinson and Ready, 1987). Three subsequent features of differentiation, rhabdomere diameter, opsin type, and axon projection pattern, which are normally selected coordinately, with a fixed association in R1-R6, large rhabdomeres, Rh1 expression, and lamina synaptic terminals, become mixed independently in transformed R1-R6 neurons.

Several examples of switched photoreceptor fates are already known among photoreceptors, with the clearest cases among the two central cells, R7 and R8. These have long been recognized to comprise either pale or a more common yellow subtype (Franceschini et al., 1981): pale ommatidia containing Rh3 in R7 and Rh5 in R8, and yellow with Rh4 in R7 in combination with Rh6 in R8 (Wernet and Desplan, 2004). This obligatory pairing arises from a signal originating in R7 (Chou et al., 1996; Papatsenko et al., 1997; Chou et al., 1999), with warts and melted reciprocally regulating the fate in R8 (Mikeladze-Dvali et al., 2005). A mutation in either warts (Rh6) or melted (Rh5) changes the opsin expressed in R8, which then fails to coordinate with the overlying R7. In either type, both cells invariably have the same rhabdomere diameter regardless of the opsin expressed. In a second example, R7 and $\mathrm{R} 8$ cells both express Rh3 in the dorsal rim area of the compound eye, as specified by the gene homothorax. Ommatidia in the dorsal rim area also undergo a decoupling between rhabdomere size and opsin expression, with the central rhabdomeres being markedly enlarged compared with R7s outside the dorsal rim area (Tomlinson, 2003; Wernet et al., 2003). Third, photoreceptor classes are distinguished by their opsin expression, which is in turn dependant on homeodomain binding sites in the opsin promoters. Orthodenticle (otd) binds these promoter sequences, and in its absence Rh3 and Rh5 expression are lost. Furthermore, in otd mutants, Rh1 expression expands to R7 and R8, whereas Rh6 expression expands to R1-R6 (Tahayato et al., 2003). We did not observe expansion of Rh1 into R7 or R8 in runtoverexpressing flies, but Rh3 and Rh6 expression were expanded to R1-R6. In the fourth example, from frontal ommatidia of the so-called love spot in male houseflies, Musca domestica, transformed R7 cells instead of projecting to the medulla terminate in the lamina, where they form a synaptic terminal like that of R1-R6 (Hardie, 1983). Our findings further exemplify that the genetic regulation of photoreceptor phenotype allows features of R1-R6 to become mixed with those of R7 and R8. An important aspect of our findings is not only that the normal coordinate expression of rhabdomere size, opsin expression, and axon projection in correct combinations is perturbed, but also that the novel combinations of such features are variable. Thus, some R1-R6 with small rhabdomeres still express Rh1, whereas some Rh1-expressing photoreceptors project to the medulla.

It remains to be seen how runt overexpression in the retina causes these changes in photoreceptor cell fate, either in opsin expression, rhabdomeres size, or terminal location. What is known, however, is that Sev expression is limited to photoreceptors R1, R3, R4, R6, and R7 during development (Banerjee et al., 1987; Tomlinson et al., 1987) and that in seven-up mutants these photoreceptors are transformed to R7 (Mlodzik et al., 1990). Thus, it is not surprising that in runt overexpression mutants it is these outer photoreceptors that are most likely to adopt features characteristic of R7 cells, such as small rhabdomeres and Rh3 expression.

For the axons of R1-R6, the transformation after runt overexpression in the eye is variable. The axons resemble those of R7 and R8 in innervating the medulla but at least some may differ in continuing to innervate lamina cells, which R7 and R8 never normally do (Meinertzhagen and O'Neil, 1991). Whereas some photoreceptor axons expand to form synaptic terminals in the lamina, others may simply form multiple synaptic zones along their length, including along extensions into the medulla. Indeed, precedents exist in other insect visual systems for long visual fiber axons, equivalent to R7 and R8 in the fly, that project to the lamina but nonetheless form synapses en passant with monopolar cells in the lamina, as in the dragonfly Sympetrum (Meinertzhagen and Armett-Kibel, 1982; Armett-Kibel and Meinertzhagen, 1985).

\section{Supernumerary photoreceptor terminals form ectopic synapses with novel targets in the medulla}

Terminals of Rh1-expressing R1-R6 photoreceptors that innervate the medulla form synapses, as exhibited by their expression of the synaptic protein Bruchpilot. In addition, supernumerary photoreceptor terminals in the medulla, which must contain terminals from many transformed R1-R6, form synapses that share many features of the tetrad synapses formed by R1-R6 terminals in the lamina. This is surprising, because at least many of the target neurons must be medulla neurons, which are foreign, and this must certainly be true beyond stratum M5, the deepest termination of L1. Although some target neurons could be the terminals of lamina cells (L1, L2, etc., the normal targets of R1-R6 in the lamina), in the wild type these terminals do not form dendrites (Fischbach and Dittrich, 1989) and are predominantly presynaptic in the medulla (Takemura et al., 2008). Moreover, medulla cells are simply more numerous than lamina cells (Fischbach and Dittrich, 1989; Meinertzhagen and Sorra, 2001) and extend throughout the entire medulla depth. The participation of lamina amacrine neurons and epithelial glial cells at ec- 
topic medulla synapses is absolutely denied, because these cells never extend to the medulla.

Ectopic synaptogenesis occurs widely in different nervous systems. Neuromuscular innervation readily forms ectopic synapses in vertebrate muscles, for example, reflecting a range of phenomena regulating the size and distribution of synaptic sites (for review, see Lømo, 2003). Likewise, developing Drosophila motoneurons denied access to their normal muscle targets form stable, ectopic synapses on other muscles (Cash et al., 1992). In the cricket, sensory afferents from transplanted cerci (abdominal sensory appendages) form functional ectopic synapses on novel interneuron targets (Murphey et al., 1983). In the vertebrate retina, genetically procured degeneration of rods results in the normal rod bipolar cell targets of these cells accepting ectopic synapses from cones (Peng et al., 2000), by a process that entails the retraction of rod terminals and neurite outgrowth from rod bipolar cell dendrites (Bayley and Morgans, 2007). Likewise, loss of cones causes cone bipolar cells to form ectopic synapses with rods, a switch that requires the presynaptic photoreceptors to be functional (Haverkamp et al., 2006), unlike the fly, in which tetrad synaptogenesis is activity independent (Hiesinger et al., 2006).

These examples of ectopic synaptogenesis all occur in response to some loss of input or target sites. Our findings now show that when their axons are redirected to a novel territory, sensory neurons such as R1-R6 can nevertheless form synapses with their normal targets, which are still intact, as well as with novel partners in the second territory. The terminals thus act with autonomy in each neuropile. The ability of photoreceptors to form ectopic synapses in foreign neuropiles has also recently been demonstrated for ectopic eyes on the antennae and legs of Drosophila, which extend axons and synapse at superficial locations in the CNS (Clements et al., 2008). In either case, the ability of such postsynaptic sites to respond to neurotransmitter released from ectopic R1-R6 terminals is of course questionable; we would predict that only the normal partners of R7 or R8 would express histamine receptors (Witte et al., 2002) and thus be able to respond to the histamine release.

\section{The presynaptic terminal of R1-R6 determines the architecture of its synaptic organelles}

Additional evidence of R1-R6 terminal autonomy comes from the more detailed examination of its synaptic organelles. Several features of the latter reveal that the size, structure, and composition of the organelles are highly conserved, regardless of the identity of the postsynaptic target cells. These features include at the release sites: the presynaptic T-bar ribbon and the quadripartite composition of its postsynaptic ensemble; and at the capitate projection, the diameter of the head and its invagination by a glial cell process. All of these features are determined by the presynaptic photoreceptor neuron, in an autonomy that confirms many other details of tetrad synaptogenesis in the lamina.

\section{References}

Armett-Kibel C, Meinertzhagen IA (1985) The long visual fibers of the dragonfly optic lobe: their cells of origin and lamina connections. J Comp Neurol 242:459-474.

Banerjee U, Renfranz PJ, Hinton DR, Rabin BA, Benzer S (1987) The sevenless + protein is expressed apically in cell membranes of developing Drosophila retina; it is not restricted to cell R7. Cell 51:151-158.

Bayley PR, Morgans CW (2007) Rod bipolar cells and horizontal cells form displaced synaptic contacts with rods in the outer nuclear layer of the nob2 retina. J Comp Neurol 500:286-298.

Braitenberg V (1967) Patterns of projection in the visual system of the fly. I. Retina-lamina projections. Exp Brain Res 3:271-298.
Brand AH, Perrimon N (1993) Targeted gene expression as a means of altering cell fates and generating dominant phenotypes. Development 118:401-415.

Cagan RL, Krämer H, Hart AC, Zipursky SL (1992) The bride of sevenless and sevenless interaction: internalization of a transmembrane ligand. Cell 69:393-399.

Campbell G, Göring H, Lin T, Spana E, Andersson S, Doe CQ, Tomlinson A (1994) RK2, a glial-specific homeodomain protein required for embryonic nerve cord condensation and viability in Drosophila. Development 120:2957-2966.

Cash S, Chiba A, Keshishian H (1992) Alternate neuromuscular target selection following the loss of single muscle fibers in Drosophila. J Neurosci 12:2051-2064.

Chamberlain LH, Burgoyne RD (2000) Cysteine-string protein: the chaperone at the synapse. J Neurochem 74:1781-1789.

Chklovskii DB, Koulakov AA (2004) Maps in the brain: what can we learn from them? Annu Rev Neurosci 27:369-392.

Choe KM, Prakash S, Bright A, Clandinin TR (2006) Liprin- $\alpha$ is required for photoreceptor target selection in Drosophila. Proc Natl Acad Sci U S A 103:11601-11606.

Chotard C, Salecker I (2004) Neurons and glia: team players in axon guidance. Trends Neurosci 27:655-661.

Chou WH, Hall KJ, Wilson DB, Wideman CL, Townson SM, Chadwell LV, Britt SG (1996) Identification of a novel Drosophila opsin reveals specific patterning of the R7 and R8 photoreceptor cells. Neuron 17:1101-1115.

Chou WH, Huber A, Bentrop J, Schulz S, Schwab K, Chadwell LV, Paulsen R, Britt SG (1999) Patterning of the R7 and R8 photoreceptor cells of Drosophila: evidence for induced and default cell-fate specification. Development 126:607-616.

Clements J, Lu Z, Gehring WJ, Meinertzhagen IA, Callaerts P (2008) Central projections of photoreceptor axons originating from ectopic eyes in Drosophila. Proc Natl Acad Sci U S A 105:8968-8973.

Dormand EL, Brand AH (1998) Runt determines cell fates in the Drosophila embryonic CNS. Development 125:1659-1667.

Dütting D, Handwerker C, Drescher U (1999) Topographic targeting and pathfinding errors of retinal axons following overexpression of ephrinA ligands on retinal ganglion cell axons. Dev Biol 216:297-311.

Evans GJ, Morgan A, Burgoyne RD (2003) Tying everything together: the multiple roles of cysteine string protein (CSP) in regulated exocytosis. Traffic 4:653-659.

Fabian-Fine R, Verstreken P, Hiesinger PR, Horne JA, Kostyleva R, Zhou Y, Bellen HJ, Meinertzhagen IA (2003) Endophilin promotes a late step in endocytosis at glial invaginations in Drosophila photoreceptor terminals. J Neurosci 23:10732-10744.

Fischbach K-F, Dittrich APM (1989) The optic lobe of Drosophila melanogaster. Part I. A Golgi analysis of wild-type structure. Cell Tissue Res 258:441-475.

Franceschini N, Kirschfeld K, Minke B (1981) Fluorescence of photoreceptor cells observed in vivo. Science 213:1264-1267.

Freeman M (1996) Reiterative use of the EGF receptor triggers differentiation of all cell types in the Drosophila eye. Cell 87:651-660.

Freeman MR (2006) Sculpting the nervous system: glial control of neuronal development. Curr Opin Neurobiol 16:119-125.

Fröhlich A, Meinertzhagen IA (1982) Synaptogenesis in the first optic neuropile of the fly's visual system. J Neurocytol 11:159-180.

Fryxell KJ, Meyerowitz EM (1987) An opsin gene that is expressed only in the R7 photoreceptor cell of Drosophila. EMBO J 6:443-451.

Górska-Andrzejak J, Stowers RS, Borycz J, Kostyleva R, Schwarz TL, Meinertzhagen IA (2003) Mitochondria are redistributed in Drosophila photoreceptors lacking milton, a kinesin-associated protein. J Comp Neurol 463:372-388.

Graham RC Jr, Karnovsky MJ (1966) The early stages of absorption of injected horseradish peroxidase in the proximal tubules of mouse kidney: ultrastructural cytochemistry by a new technique. J Histochem Cytochem 14:291-302.

Grenningloh G, Rehm EJ, Goodman CS (1991) Genetic analysis of growth cone guidance in Drosophila: fasciclin II functions as a neuronal recognition molecule. Cell 67:45-57.

Halter DA, Urban J, Rickert C, Ner SS, Ito K, Travers AA, Technau GM (1995) The homeobox gene repo is required for the differentiation and 
maintenance of glia function in the embryonic nervous system of Drosophila melanogaster. Development 121:317-332.

Hardie RC (1983) Projection and connectivity of sex-specific photoreceptors in the compound eye of the male housefly (Musca domestica). Cell Tissue Res 233:1-21.

Hardie RC (1987) Is histamine a neurotransmitter in insect photoreceptors? J Comp Physiol A Neuroethol Sens Neural Behav Physiol 161:201-213.

Hardie RC, Kirschfeld K (1983) Ultraviolet sensitivity of fly photoreceptors R7 and R8: evidence for a sensitizing function. Biophys Struct Mech 9:171-180.

Haverkamp S, Michalakis S, Claes E, Seeliger MW, Humphries P, Biel M, Feigenspan A (2006) Synaptic plasticity in $\mathrm{CNGA3}^{-1-}$ mice: cone bipolar cells react on the missing cone input and form ectopic synapses with rods. J Neurosci 26:5248-5255.

Hiesinger PR, Reiter C, Schau H, Fischbach KF (1999) Neuropil pattern formation and regulation of cell adhesion molecules in Drosophila optic lobe development depend on synaptobrevin. J Neurosci 19:7548-7556.

Hiesinger PR, Zhai RG, Zhou Y, Koh TW, Mehta SQ, Schulze KL, Cao Y, Verstreken P, Clandinin TR, Fischbach KF, Meinertzhagen IA, Bellen HJ (2006) Activity-independent prespecification of synaptic partners in the visual map of Drosophila. Curr Biol 16:1835-1843.

Hing H, Xiao J, Harden N, Lim L, Zipursky SL (1999) Pak functions downstream of Dock to regulate photoreceptor axon guidance in Drosophila. Cell 97:853-863.

Huang Z, Kunes S (1996) Hedgehog, transmitted along retinal axons, triggers neurogenesis in the developing visual centers of the Drosophila brain. Cell 86:411-422.

Huang Z, Kunes S (1998) Signals transmitted along retinal axons in Drosophila: Hedgehog signal reception and the cell circuitry of lamina cartridge assembly. Development 125:3753-3764.

Huang Z, Shilo BZ, Kunes S (1998) A retinal axon fascicle uses spitz, an EGF receptor ligand, to construct a synaptic cartridge in the brain of Drosophila. Cell 95:693-703.

Johnson DA, Donovan SL, Dyer MA (2006) Mosaic deletion of $R b$ arrests rod differentiation and stimulates ectopic synaptogenesis in the mouse retina. J Comp Neurol 498:112-128.

Johnson KG, McKinnell IW, Stoker AW, Holt CE (2001) Receptor protein tyrosine phosphatases regulate retinal ganglion cell axon outgrowth in the developing Xenopus visual system. J Neurobiol 49:99-117.

Jones B, McGinnis W (1993) A new Drosophila homeobox gene, bsh, is expressed in a subset of brain cells during embryogenesis. Development 117:793-806.

Kaas JH (1997) Topographic maps are fundamental to sensory processing. Brain Res Bull 44:107-112.

Kaminker JS, Canon J, Salecker I, Banerjee U (2002) Control of photoreceptor axon target choice by transcriptional repression of Runt. Nat Neurosci 5:746-750.

Kirschfeld K (1967) Die Projektion der optischen Umwelt auf das Raster der Rhabdomere im Komplexauge von MUSCA. Exp Brain Res 3:248-270.

Kittel RJ, Wichmann C, Rasse TM, Fouquet W, Schmidt M, Schmid A, Wagh DA, Pawlu C, Kellner RR, Willig KI, Hell SW, Buchner E, Heckmann M, Sigrist SJ (2006) Bruchpilot promotes active zone assembly, $\mathrm{Ca}^{2+}$ channel clustering, and vesicle release. Science 312:1051-1054.

Koushika SP, Lisbin MJ, White K (1996) ELAV, a Drosophila neuronspecific protein, mediates the generation of an alternatively spliced neural protein isoform. Curr Biol 6:1634-1641.

Larsen CW, Hirst E, Alexandre C, Vincent JP (2003) Segment boundary formation in Drosophila embryos. Development 130:5625-5635.

Laughlin SB, Howard J, Blakeslee B (1987) Synaptic limitations to contrast coding in the retina of the blowfly Calliphora. Proc R Soc Lond B Biol Sci 231:437-467.

Lee T, Luo L (1999) Mosaic analysis with a repressible cell marker for studies of gene function in neuronal morphogenesis. Neuron 22:451-461.

Lømo T (2003) What controls the position, number, size, and distribution of neuromuscular junctions on rat muscle fibers? J Neurocytol $32: 835-848$.

Mardon G, Solomon NM, Rubin GM (1994) dachshund encodes a nuclear protein required for normal eye and leg development in Drosophila. Development 120:3473-3486.

Mast JD, Prakash S, Chen PL, Clandinin TR (2006) The mechanisms and molecules that connect photoreceptor axons to their targets in Drosophila. Semin Cell Dev Biol 17:42-49.
McLaughlin T, O'Leary DDM (2005) Molecular gradients and development of retinotopic maps. Annu Rev Neurosci 28:327-355.

Meinertzhagen IA, Armett-Kibel C (1982) The lamina monopolar cells in the optic lobe of the dragonfly Sympetrum. Philos Trans R Soc Lond B Biol Sci 297:27-49.

Meinertzhagen IA, Hanson TE (1993) The development of the optic lobe. In: The development of Drosophila melanogaster (Bate M, Martinez-Arias A, eds), pp 1363-1491. Cold Spring Harbor, NY: Cold Spring Harbor Press.

Meinertzhagen IA, O’Neil SD (1991) Synaptic organization of columnar elements in the lamina of the wild type in Drosophila melanogaster. J Comp Neurol 305:232-263.

Meinertzhagen IA, Sorra KE (2001) Synaptic organization in the fly's optic lamina: few cells, many synapses and divergent microcircuits. Prog Brain Res 131:53-69.

Mikeladze-Dvali T, Wernet MF, Pistillo D, Mazzoni EO, Teleman AA, Chen YW, Cohen S, Desplan C (2005) The growth regulators warts/lats and melted interact in a bistable loop to specify opposite fates in Drosophila R8 photoreceptors. Cell 122:775-787.

Mlodzik M, Hiromi Y, Weber U, Goodman CS, Rubin GM (1990) The Drosophila seven-up gene, a member of the steroid receptor gene superfamily, controls photoreceptor cell fates. Cell 60:211-224.

Montell C, Jones K, Zuker C, Rubin G (1987) A second opsin gene expressed in the ultraviolet-sensitive R7 photoreceptor cells of Drosophila melanogaster. J Neurosci 7:1558-1566.

Morante J, Desplan C (2004) Building a projection map for photoreceptor neurons in the Drosophila optic lobes. Semin Cell Dev Biol 15:137-143.

Moses K, Rubin GM (1991) glass encodes a site-specific DNA-binding protein that is regulated in response to positional signals in the developing Drosophila eye. Genes Dev 5:583-593.

Murphey RK, Bacon JP, Sakaguchi DS, Johnson SE (1983) Transplantation of cricket sensory neurons to ectopic locations: arborizations and synaptic connections. J Neurosci 3:659-672.

Nicol D, Meinertzhagen IA (1982) An analysis of the number and composition of the synaptic populations formed by photoreceptors of the fly. J Comp Neurol 207:29-44.

O’Tousa JE, Baehr W, Martin RL, Hirsh J, Pak WL, Applebury ML (1985) The Drosophila ninaE gene encodes an opsin. Cell 40:839-850.

Papatsenko D, Sheng G, Desplan C (1997) A new rhodopsin in R8 photoreceptors of Drosophila: evidence for coordinate expression with Rh3 in R7 cells. Development 124:1665-1673.

Peng YW, Hao Y, Petters RM, Wong F (2000) Ectopic synaptogenesis in the mammalian retina caused by rod photoreceptor-specific mutations. Nat Neurosci 3:1121-1127.

Peng YW, Senda T, Hao Y, Matsuno K, Wong F (2003) Ectopic synaptogenesis during retinal degeneration in the royal college of surgeons rat. Neuroscience 119:813-820.

Poeck B, Fischer S, Gunning D, Zipursky SL, Salecker I (2001) Glial cells mediate target layer selection of retinal axons in the developing visual system of Drosophila. Neuron 29:99-113.

Prokop A, Meinertzhagen IA (2006) Development and structure of synaptic contacts in Drosophila. Semin Cell Dev Biol 17:20-30.

Rao-Mirotznik R, Harkins AB, Buchsbaum G, Sterling P (1995) Mammalian rod terminal: architecture of a binary synapse. Neuron 14:561-569.

Richardt A, Rybak J, Störtkuhl KF, Meinertzhagen IA, Hovemann BT (2002) Ebony protein in the Drosophila nervous system: optic neuropile expression in glial cells. J Comp Neurol 452:93-102.

Robinow S, White K (1991) Characterization and spatial distribution of the ELAV protein during Drosophila melanogaster development. J Neurobiol 22:443-461.

Salcedo E, Huber A, Henrich S, Chadwell LV, Chou WH, Paulsen R, Britt SG (1999) Blue- and green-absorbing visual pigments of Drosophila: ectopic expression and physiological characterization of the R8 photoreceptor cell-specific Rh5 and Rh6 rhodopsins. J Neurosci 19:10716-10726.

Selleck SB, Gonzalez C, Glover DM, White K (1992) Regulation of the $\mathrm{G}_{1}-\mathrm{S}$ transition in postembryonic neuronal precursors by axon ingrowth. Nature 355:253-255.

Spana EP, Doe CQ (1995) The prospero transcription factor is asymmetrically localized to the cell cortex during neuroblast mitosis in Drosophila. Development 121:3187-3195.

Tahayato A, Sonneville R, Pichaud F, Wernet MF, Papatsenko D, Beaufils P, 
Cook T, Desplan C (2003) Otd/Crx, a dual regulator for the specification of ommatidia subtypes in the Drosophila retina. Dev Cell 5:391-402.

Takemura SY, Lu Z, Meinertzhagen IA (2008) Synaptic circuits of the Drosophila optic lobe: the input terminals to the medulla. J Comp Neurol 509:493-513.

Tissot M, Gendre N, Hawken A, Störtkuhl KF, Stocker RF (1997) Larval chemosensory projections and invasion of adult afferents in the antennal lobe of Drosophila. J Neurobiol 32:281-297.

Tix S, Eule E, Fischbach KF, Benzer S (1997) Glia in the chiasms and medulla of the Drosophila melanogaster optic lobes. Cell Tissue Res 289:397-409.

Tomlinson A (2003) Patterning the peripheral retina of the fly: decoding a gradient. Dev Cell 5:799-809.

Tomlinson A, Ready DF (1987) Neuronal differentiation in the Drosophila ommatidium. Dev Biol 120:366-376.

Tomlinson A, Bowtell DD, Hafen E, Rubin GM (1987) Localization of the sevenless protein, a putative receptor for positional information, in the eye imaginal disc of Drosophila. Cell 51:143-150.

Townson SM, Chang BS, Salcedo E, Chadwell LV, Pierce NE, Britt SG (1998) Honeybee blue- and ultraviolet-sensitive opsins: cloning, heterologous expression in Drosophila, and physiological characterization. J Neurosci 18:2412-2422.

Trujillo-Cenóz O (1965) Some aspects of the structural organization of the intermediate retina of dipterans. J Ultrastruct Res 13:1-33.

Van Vactor D Jr, Krantz DE, Reinke R, Zipursky SL (1988) Analysis of mutants in chaoptin, a photoreceptor cell-specific glycoprotein in Drosophila, reveals its role in cellular morphogenesis. Cell 52:281-290.
Wagh DA, Rasse TM, Asan E, Hofbauer A, Schwenkert I, Dürrbeck H, Buchner S, Dabauvalle MC, Schmidt M, Qin G, Wichmann C, Kittel R, Sigrist SJ, Buchner E (2006) Bruchpilot, a protein with homology to ELKS/ CAST, is required for structural integrity and function of synaptic active zones in Drosophila. Neuron 49:833-844.

Wernet MF, Desplan C (2004) Building a retinal mosaic: cell-fate decision in the fly eye. Trends Cell Biol 14:576-584.

Wernet MF, Labhart T, Baumann F, Mazzoni EO, Pichaud F, Desplan C (2003) Homothorax switches function of Drosophila photoreceptors from color to polarized light sensors. Cell 115:267-279.

Witte I, Kreienkamp HJ, Gewecke M, Roeder T (2002) Putative histaminegated chloride channel subunits of the insect visual system and thoracic ganglion. J Neurochem 83:504-514.

Zinsmaier KE, Hofbauer A, Heimbeck G, Pflugfelder GO, Buchner S, Buchner E (1990) A cysteine-string protein is expressed in retina and brain of Drosophila. J Neurogenet 7:15-29.

Zinsmaier KE, Eberle KK, Buchner E, Walter N, Benzer S (1994) Paralysis and early death in cysteine string protein mutants of Drosophila. Science 263:977-980.

Zipursky SL, Venkatesh TR, Teplow DB, Benzer S (1984) Neuronal development in the Drosophila retina: monoclonal antibodies as molecular probes. Cell 36:15-26.

Zuker CS, Montell C, Jones K, Laverty T, Rubin GM (1987) A rhodopsin gene expressed in photoreceptor cell R7 of the Drosophila eye: homologies with other signal-transducing molecules. J Neurosci 7:1550-1557. 